\title{
Spectroscopy, Photophysical Properties and Crystal Structures of Luminescent Diplatinum(1) Complexes of Bis(diphenylphosphino)methane $\dagger$
}

\author{
Hon-Kay Yip, ${ }^{a}$ Chi-Ming Che ${ }^{*, a}$ and Shie-Ming Peng ${ }^{b}$ \\ a Department of Chemistry. University of Hong Kong, Pokfulam Road, Hong Kong \\ ${ }^{b}$ Department of Chemistry, National Taiwan University, Taipei, Taiwan
}

\begin{abstract}
The reactions of quinoline (quin), 1 -methylimidazole (mim), 4-tert-butylpyridine (4Bu'-py) and 2,4,6trimethylpyridine (tmpy) with $\left[\mathrm{Pt}_{2}(\mathrm{dppm})_{2} \mathrm{Cl}_{2}\right]$ in methanol yielded $\left[\mathrm{Pt}_{2}(\mathrm{dppm})(\text { quin })_{2}\right]^{2+},\left[\mathrm{Pt}_{2}(\mathrm{dppm})_{2}\right.$ $\left.(\mathrm{mim})_{2}\right]^{2+},\left[\mathrm{Pt}_{2}(\mathrm{dppm})_{2}\left(4 \mathrm{Bu}^{\mathrm{t}}-\mathrm{py}\right)_{2}\right]^{2+}$ and $\left[\mathrm{Pt}_{2}(\mathrm{dppm})_{2}(\mathrm{tmpy}) \mathrm{Cl}\right]^{+}$, respectively. The complexes were isolated as either hexafluorophosphate or perchlorate salts. The crystallographically measured intramolecular $\mathrm{Pt}-\mathrm{Pt}$ distances are $2.615(1), 2.580(1)$ and $2.627(2) \AA$ for $\left[\mathrm{Pt}_{2}(\mathrm{dppm})_{2}(\text { quin })_{2}\right]^{2+}$, $\left[\mathrm{Pt}_{2}(\mathrm{dppm})_{2}(\mathrm{mim})_{2}\right]^{2+}$ and $\left[\mathrm{Pt}_{2}(\mathrm{dppm})_{2}(\mathrm{tmpy}) \mathrm{Cl}\right]^{+}$respectively, indicating the presence of a single $\mathrm{Pt}-\mathrm{Pt}$ bond. The ${ }^{1} \mathrm{H}$ and ${ }^{31} \mathrm{P}$ NMR spectra of the complexes have been recorded. Solution UV/VIS spectra of the four new complexes as well as those of $\left[\mathrm{Pt}_{2}(\mathrm{dppm})_{2}\left(\mathrm{PPh}_{3}\right)_{2}\right]\left[\mathrm{PF}_{6}\right]_{2}$ and $\left[\mathrm{Pt}_{2}(\mathrm{dppm})_{2}(\mathrm{py})_{2}\right]\left[\mathrm{PF}_{6}\right]_{2}$ (py $=$ pyridine) were recorded. The lowest-energy absorption band is assigned to $a^{1}\left(d_{\pi^{*}} \longrightarrow d_{\sigma^{*}}\right)$ transition. All of the diplatinum(1) complexes display solid-state luminescence at room temperature and at $77 \mathrm{~K}$ with long emission lifetimes.
\end{abstract}

Dinuclear complexes containing metal-metal bonds continue to play a crucial role in the development of inorganic and organometallic photochemistry. Much attention has been paid to the $d^{7}-d^{7}$ systems because of their novel photochemistry, spectroscopic properties and structural features. For example, the photodissociation of the well celebrated unbridged $\left[\mathrm{Mn}_{2}-\right.$ $\left.(\mathrm{CO})_{10}\right]^{1}$ has been studied and the electronic absorption and Raman spectroscopy of $\left[\mathrm{Rh}_{2}{ }_{2}\left(\mathrm{O}_{2} \mathrm{CR}\right)_{4} \mathrm{~L}_{2}\right]^{2}$ and $\left[\mathrm{Pt}^{\mathrm{III}}{ }_{2^{-}}\right.$ $\left.\left(\mathrm{H}_{2} \mathrm{P}_{2} \mathrm{O}_{5}\right)_{4} \mathrm{~L}_{2}\right]^{n-} \mathrm{L}=$ axial ligand $)^{3}$ have been extensively investigated. The study of the luminescent behaviour of the $\mathrm{d}^{7}-$ $\mathrm{d}^{7}$ systems was previously hindered by the fact that the efficient photodissociation process arising from the $d_{\sigma} \longrightarrow d_{\sigma^{*}}$ transition renders unbridged dimers like $\left[\mathrm{Mn}_{2}(\mathrm{CO})_{10}\right]$ or $\left[\operatorname{Re}_{2}(\mathrm{CO})_{10}\right]^{4}$ non-emissive. However, Stiegman et al. ${ }^{5}$ have suggested the use of a bridging ligand like $\mathrm{H}_{2} \mathrm{P}_{2} \mathrm{O}_{5}{ }^{2-}$ or dppm [bis(diphenylphosphino)methane] to prevent the dissociation and these workers have studied the luminescent properties of $\left[\mathrm{Pt}_{2}\left(\mathrm{H}_{2} \mathrm{P}_{2} \mathrm{O}_{5}\right)_{4} \mathrm{~L}_{2}\right]^{4-}(\mathrm{L}=\mathrm{Cl}, \mathrm{Br}, \mathrm{SCN}$ or pyridine $)$. Subsequent studies have been made on the electronic absorption and emission spectroscopy of other $d^{7}-d^{7}$ systems like $\left[\operatorname{Re}_{2}(\mathrm{dmpm})_{2}(\mathrm{CO})_{6}\right]^{6} \quad[\mathrm{dmpm}=$ bis(dimethylphosphino)methane], $\left[\mathrm{Pt}_{2}\left(\mathrm{HPG}_{4}\right)_{4} \mathrm{~L}_{2}\right]^{n-7}$ and $\left[\mathrm{Rh}_{2}\{\mathrm{NMe}-\right.$ $\left.\left.\left(\mathrm{PF}_{3}\right)_{2}\right\}_{3} \mathrm{Cl}_{4}\right]^{8}$ and of the $\mathrm{d}^{7}-\mathrm{d}^{9}$ complex $\left[\mathrm{Rh}_{2}\{\mathrm{NMe}-\right.$ $\left.\left.\left(\mathrm{PF}_{3}\right)_{2}\right\}_{3} \mathrm{Cl}_{2}\left(\mathrm{PF}_{3}\right)\right]^{8}$. Another class of dinuclear metal complexes containing a single metal-metal bond is the $d^{9}-d^{9}$ system. Dinuclear $\mathrm{Pt}^{1}$ bridged by dppm is the prototype of such complexes, ${ }^{9}$ which are well known catalysts for the activation of small molecules. It is therefore interesting to investigate the spectroscopic properties of this class of dinuclear complexes. Herein we describe the syntheses, structural characterization, electronic absorption and emission spectroscopy of several dinuclear complexes $\left[\mathrm{Pt}_{2}(\mathrm{dppm})_{2} \mathrm{X}(\mathrm{Y})\right]^{n+}[n=2, \mathrm{X}=\mathrm{Y}=$ $\mathrm{PPh}_{3}, \operatorname{mim}(1-$ methylimidazole), quin(quinoline), $\mathrm{py}$ (pyridine) or 4But-py(4-tert-butylpyridine); $n=1, \mathrm{X}=\operatorname{tmpy}(2,4,6$-trimethylpyridine), $\mathrm{Y}=\mathrm{Cl}]$.

† Supplementary data available: see Instructions for Authors, J. Chem. Soc., Dalton Trans., 1993, Issue 1, pp. xxiii-xxviii.

\section{Experimental}

Synthesis of Metal Complexes.-The complexes $\left[\mathrm{Pt}_{2}(\mathrm{dppm})_{2}-\right.$ $\left.\left(\mathrm{PPh}_{3}\right)_{2}\right]\left[\mathrm{PF}_{6}\right]_{2}$ and $\left[\mathrm{Pt}_{2}(\mathrm{dppm})_{2}(\mathrm{py})_{2}\right]\left[\mathrm{PF}_{6}\right]_{2}$ were prepared according to the published method. ${ }^{10} \mathrm{~A}$ similar method ${ }^{10}$ was also employed for the synthesis of $\left[\mathrm{Pt}_{2}(\mathrm{dppm})_{2}(\text { quin })_{2}\right]\left[\mathrm{PF}_{6}\right]_{2}$, $\left[\mathrm{Pt}_{2}(\mathrm{dppm})_{2}(\mathrm{mim})_{2}\right] \mathrm{X}_{2}\left(\mathrm{X}=\mathrm{ClO}_{4}\right.$ or $\left.\mathrm{PF}_{6}\right),\left[\mathrm{Pt}_{2}(\mathrm{dppm})_{2^{-}}\right.$ $\left.\left(4 \mathrm{Bu}^{\mathrm{t}}-\mathrm{py}\right)_{2}\right]\left[\mathrm{PF}_{6}\right]_{2}$ and $\left[\mathrm{Pt}_{2}(\mathrm{dppm})_{2}(\mathrm{tmpy}) \mathrm{Cl}\right] \mathrm{PF}_{6}$. These were prepared by adding an excess of the axial nitrogen base ligand to a suspension of $\left[\mathrm{Pt}_{2}(\mathrm{dppm})_{2} \mathrm{Cl}_{2}\right]$ in methanol; the mixture was stirred until a clear solution was obtained. The solution was then filtered and the complex cation precipitated by adding either $\mathrm{NH}_{4} \mathrm{PF}_{6}$ or $\mathrm{LiClO}_{4}$ to the filtrate. Adding stoichiometric or excess amounts of tmpy gave the complex $\left[\mathrm{Pt}_{2}(\mathrm{dppm})_{2}\right.$ (tmpy)Cl $]^{+}$as the only isolable product (yield $65-72 \%$ ) \{Found: C, 51.5; H, 3.6. Calc. for $\left[\mathrm{Pt}_{2}(\mathrm{dppm})_{2}\left(\mathrm{PPh}_{3}\right)_{2}\right]\left[\mathrm{PF}_{6}\right]_{2}$ : $\mathrm{C}, 52.3 ; \mathrm{H}, 3.8$. Found: $\mathrm{C}, 44.3 ; \mathrm{H}, 3.2 ; \mathrm{N}, 1.8$. Calc. for $\left[\mathrm{Pt}_{2}(\mathrm{dppm})_{2}(\mathrm{py})_{2}\right]\left[\mathrm{PF}_{6}\right]_{2}: \mathrm{C}, 44.8 ; \mathrm{H}, 3.4 ; \mathrm{N}, 1.7$. Found: $\mathrm{C}$, $46.2 ; \mathrm{H}, 3.2 ; \mathrm{N}, \quad 1.4$. Calc. for $\left.\left[\mathrm{Pt}_{2}(\mathrm{dppm})_{2} \text { (quin) }\right)_{2}\right]-$ $\left[\mathrm{PF}_{6}\right]_{2} \cdot 2 \mathrm{CH}_{2} \mathrm{Cl}_{2}: \mathrm{C}, 46.2 ; \mathrm{H}, 3.4 ; \mathrm{N}, 1.55$. Found: $\mathrm{C}, 47.2 ; \mathrm{H}, 3.8$; $\mathrm{N}, 1.6$. Calc. for $\left[\mathrm{Pt}_{2}(\mathrm{dppm})_{2}(4 \mathrm{Bu} \text { '-py })_{2}\right]\left[\mathrm{PF}_{6}\right]_{2}: \mathrm{C}, 47.5 ; \mathrm{H}, 4.1$; $\mathrm{N}$, 1.6. Found: $\mathrm{C}, 42.6 ; \mathrm{H}, 3.3 ; \mathrm{N}, 3.5$. Calc. for $\left[\mathrm{Pt}_{2}-\right.$ $\left.(\mathrm{dppm})_{2}(\mathrm{mim})_{2}\right]\left[\mathrm{PF}_{6}\right]_{2}: \mathrm{C}, 43.2 ; \mathrm{H}, 3.5 ; \mathrm{N}, 3.5$. Found: C, 47.8; $\mathrm{H}, 3.8 ; \mathrm{N}, 1.0$. Calc. for $\left[\mathrm{Pt}_{2}(\mathrm{dppm})_{2}(\mathrm{tmpy}) \mathrm{Cl}\right] \mathrm{PF}_{6} \cdot \mathrm{Me}_{2} \mathrm{CO}: \mathrm{C}$, $48.2 ; \mathrm{H}, 4.0 ; \mathrm{N}, 0.9 \%\}$.

Spectroscopic Measurements.-Electronic absorption spectra were recorded on a Milton Roy Spectronic 3000 array spectrometer, emission spectra at room temperature and $77 \mathrm{~K}$ on a Spex Fluorolog-2 spectrofluorometer. The emission lifetimes were measured by using a Spectra-Physics DCR-3 Nd:YAG pulsed laser with signal acquired by a Tektronix 2430 instrument. The ${ }^{31} \mathrm{P}$ and ${ }^{1} \mathrm{H}$ NMR spectra were recorded on a $270 \mathrm{MHz}$ JEOL JMN-GSX 270 spectrometer.

$X$-Ray Structure Determination.-Crystals of $\left[\mathrm{Pt}_{2}(\mathrm{dppm})_{2}-\right.$ (quin) $\left.)_{2}\right]\left[\mathrm{PF}_{6}\right]_{2},\left[\mathrm{Pt}(\mathrm{dppm})_{2}(\mathrm{tmpy}) \mathrm{Cl}\right] \mathrm{PF}_{6}$ and $\left[\mathrm{Pt}_{2}(\mathrm{dppm})_{2}-\right.$ $\left.(\mathrm{mim})_{2}\right]\left[\mathrm{ClO}_{4}\right]_{2}$ were obtained by vapour diffusion of diethyl ether into dichloromethane, acetone and acetonitrile solution of the complexes, respectively.

Details of crystal parameters, data collection, and structure 
Table 1 Crystallographic details for $\left[\mathrm{Pt}_{2}(\mathrm{dppm})_{2}(\mathrm{quin})_{2}\right]\left[\mathrm{PF}_{6}\right]_{2} \cdot 2 \mathrm{CH}_{2} \mathrm{Cl}_{2} \mathbf{1},\left[\mathrm{Pt}_{2}(\mathrm{dppm})_{2}(\mathrm{tmpy}) \mathrm{Cl}\right] \mathrm{PF}_{6} \cdot \mathrm{Me}_{2} \mathrm{CO} 2$ and $\left[\mathrm{Pt}_{2}(\mathrm{dppm})_{2}(\mathrm{mim})_{2}\right]-$ $\left[\mathrm{ClO}_{4}\right]_{2} \cdot \mathrm{MeCN} 3$

\begin{tabular}{|c|c|c|c|}
\hline Compound & 1 & $\mathbf{2}$ & 3 \\
\hline $\begin{array}{l}\text { Empirical formula } \\
M\end{array}$ & $\begin{array}{l}\mathrm{C}_{70} \mathrm{H}_{62} \mathrm{Cl}_{4} \mathrm{~F}_{12} \mathrm{~N}_{2} \mathrm{P}_{6} \mathrm{Pt}_{2} \\
1877.4\end{array}$ & $\begin{array}{l}\mathrm{C}_{61} \mathrm{H}_{61} \mathrm{ClF}_{6} \mathrm{NOP}_{5} \mathrm{Pt}_{2} \\
1518.8\end{array}$ & $\begin{array}{l}\mathrm{C}_{60} \mathrm{H}_{59} \mathrm{Cl}_{2} \mathrm{~N}_{5} \mathrm{O}_{8} \mathrm{P}_{4} \mathrm{Pt}_{2} \\
1563.3\end{array}$ \\
\hline Crystal system & Triclinic & Monoclinic & Orthorhombic \\
\hline Space group & $P \bar{I}$ & $P 2_{1} / n$ & $\mathrm{Pca2}_{1}$ \\
\hline$a / \AA ̊ \Omega$ & $12.39(1)$ & $16.552(2)$ & $22.860(9)$ \\
\hline$b / \AA$ & $14.308(5)$ & $15.940(8)$ & $13.041(8)$ \\
\hline$c / \AA \AA$ & $21.66(1)$ & $22.614(4)$ & $20.651(7)$ \\
\hline$\alpha /^{\circ}$ & $76.64(3)$ & & \\
\hline$\beta /^{\circ}$ & $77.71(5)$ & $99.47(1)$ & \\
\hline$\gamma /^{\circ}$ & $75.83(4)$ & & \\
\hline$U / \AA^{3}$ & $3573(3)$ & $5885(3)$ & $6157(5)$ \\
\hline$z$ & 2 & 4 & 4 \\
\hline$D_{\mathrm{c}} / \mathrm{Mg} \mathrm{m}^{-3}$ & 1.746 & 1.715 & 1.687 \\
\hline$\mu / \mathrm{cm}^{-1}$ & 41.4 & 50.4 & 24.1 \\
\hline$F(000)$ & 1836 & 2976 & 2932 \\
\hline Crystal dimensions $/ \mathrm{mm}$ & $0.20 \times 0.30 \times 0.40$ & $0.05 \times 0.10 \times 0.40$ & $0.25 \times 0.30 \times 0.40$ \\
\hline Collection range, $2 \theta_{\max }{ }^{\circ}$ & $45( \pm h k \pm l)$ & $45(h k \pm l)$ & $48(h k l)$ \\
\hline Scan speed $/{ }^{\circ} \mathrm{min}^{-1}$ & $1.65-8.24$ & $1.65-8 . \overline{24}$ & $1.65-8.24$ \\
\hline Scan width $/^{\circ}$ & $0.75+0.35 \tan \theta$ & $0.75+0.35 \tan \theta$ & $0.8+0.35 \tan \theta$ \\
\hline Independent reflections & 9328 & 7669 & 4964 \\
\hline $\begin{array}{l}\text { Observed reflections, } \\
{[I>2 \sigma(I)], m}\end{array}$ & 6717 & 4261 & 3334 \\
\hline No. of parameters, $p$ & 526 & 694 & 729 \\
\hline$R^{a}$ (all reflections) & $0.053(0.081)$ & $0.049(0.117)$ & $0.037(0.081)$ \\
\hline$R^{\prime b}$ (all reflections) & $0.056(0.057)$ & $0.043(0.047)$ & $0.026(0.028)$ \\
\hline$S^{c}$ & 3.62 & 1.79 & 1.33 \\
\hline $\begin{array}{l}\text { Residual extrema in final } \\
\text { difference map } / \mathrm{e} \AA^{-3}\end{array}$ & $-1.55,+1.61$ & $-1.76,+1.23$ & $-0.60,+0.98$ \\
\hline
\end{tabular}

refinement are given in Table 1. Raw intensities were collected on a Nonius CAD4 fully automated four-circle diffractometer (graphite-monochromatized Mo-K $\alpha$ radiation) using the $\omega-2 \theta$ scan mode at $297 \mathrm{~K}$. Cell dimensions were obtained from 25 reflections with $2 \theta$ in the range $20.00-28.86^{\circ}$. All data reduction and structure refinement were performed using the NRCCSDP-VAX packages. The structure was solved by the Patterson method and refined by least squares. The $U_{i j}$ parameters of atoms in anions and solvent molecules are quite high, which is due to disorder. Tables 2 and 3 list the atomic coordinates of non-hydrogen atoms and selected bond lengths and angles respectively.

Additional material available from the Cambridge Crystallographic Data Centre comprises $\mathbf{H}$-atom coordinates, thermal parameters and remaining bond lengths and angles.

\section{Results and Discussion}

Structures of $\left[\mathrm{Pt}_{2}(\mathrm{dppm})_{2}(\text { quin })_{2}\right]\left[\mathrm{PF}_{6}\right]_{2}, \quad\left[\mathrm{Pt}_{2}(\mathrm{dppm})_{2}-\right.$ $\left.(\mathrm{mim})_{2}\right]\left[\mathrm{ClO}_{4}\right]_{2}$ and $\left[\mathrm{Pt}_{2}(\mathrm{dppm})_{2}(\mathrm{tmpy}) \mathrm{Cl} \mathrm{PF}_{6}\right.$. - In contrast to the numerous structurally characterized $\mathrm{Pt}^{\mathrm{III}}-\mathrm{Pt}^{\mathrm{III}}$ and $R h^{\text {II }}-\mathrm{Rh}^{\mathrm{Il}}$ complexes, there are only few examples of $\mathrm{Pt}^{\mathrm{I}}-\mathrm{Pt}^{\mathrm{I}}$ complexes studied by X-ray crystallography. It is expected that the intramolecular metal-metal distance may depend on the nature of the ligands (here abbreviated as L) trans to the metalmetal bond. The crystal structures of $\left.\left[\mathrm{Pt}_{2}(\mathrm{dppm})_{2} \text { (quin) }\right)_{2}\right]-$ $\left[\mathrm{PF}_{6}\right]_{2},\left[\mathrm{Pt}_{2}(\mathrm{dppm})_{2}(\mathrm{mim})_{2}\right]\left[\mathrm{ClO}_{4}\right]_{2}$ and $\left[\mathrm{Pt}_{2}(\mathrm{dppm})_{2}(\mathrm{tmpy})-\right.$ $\mathrm{Cl} \mathrm{PF}_{6}$ have been determined and the views of the cations are shown in Figs. 1, 2 and 3, respectively. Similar to other $\mathbf{P t}^{\mathbf{1}}-\mathbf{P t}^{\mathbf{1}}$ complexes of dppm, the $\mathrm{Pt}$ atoms in these three complexes adopt a more or less square-planar geometry. The L-Pt-P angles are close to $90^{\circ}$ and the L-Pt-Pt angles do not deviate much from rectilinearity. The twisted $\mathrm{Pt}_{2} \mathrm{P}_{4} \mathrm{C}_{2}$ metallocycles display a conformation which is intermediate between halfchair and envelope and this highly resembles the conformation found in $\left[\mathrm{Pt}_{2}(\mathrm{dppm})_{2}(\mathrm{CO})_{2}\right]^{2+}[$ ref. $9(c)]$ and $\left[\mathrm{Pt}_{2^{-}}\right.$ $\left.(\mathrm{dppm})_{2} \mathrm{Cl}_{2}\right]^{.9 b}$ The most important structural feature is the short Pt-Pt separation, clearly indicating the presence of a $\mathrm{Pt}-\mathrm{Pt}$ bond. Comparison with other reported structures reveals an increasing order of Pt-Pt distance: $\left[\mathrm{Pt}_{2}(\mathrm{dppm})_{2}(\mathrm{mim})_{2}\right]^{2+}$ $\left.[2.580(1)]<\left[\mathrm{Pt}_{2}(\mathrm{dppm})_{2} \text { (quin) }\right]_{2}\right]^{2}[2.615(1)]<\left[\mathrm{Pt}_{2}(\mathrm{dppm})_{2}-\right.$ (CO)Cl $]^{+}[2.620(1)]^{9 a}<\left[\mathrm{Pt}_{2}(\mathrm{dppm})_{2}(\mathrm{tmpy}) \mathrm{Cl}\right]^{+}[2.627(2)]$ $<\left[\mathrm{Pt}_{2}(\mathrm{dppm})_{2}(\mathrm{CO})_{2}\right]^{2+}[2.642(1)]^{9 c}<\left[\mathrm{Pt}_{2}(\mathrm{dppm})_{2} \mathrm{Cl}_{2}\right]$ $(2.642)^{9 b}<\left[\mathrm{Pt}_{2}(\mathrm{dppm})_{2}(\mathrm{CN})_{2}\right](2.7041 \AA){ }^{9 d}$ It is noted that the trend in Pt-Pt separation is roughly parallel with the $\sigma$-trans effect of the axial ligand. However, it is interesting to find that the trans effect exerted by the ligand tmpy is quite similar to that of carbon monoxide. A significant shortening of the Pt-Pt separation on changing the axial ligand from the weaker base quinoline to the stronger base 1-methylimidazole is also observed; as found in the complexes $\left[\mathrm{Rh}_{2}\left(\mathrm{O}_{2} \mathrm{CR}\right)_{4} \mathrm{~L}_{2}\right]{ }^{11}$ This is explained by the fact that the more conjugated heterocyclic ligand would possess more symmetrically suitable orbitals to interact with the metal centres leading to an increase in metalmetal distance. The $\mathrm{Pt}-\mathrm{N}$ (axial nitrogen base) distances of $\left[\mathrm{Pt}_{2}(\mathrm{dppm})_{2}(\mathrm{mim})_{2}\right]\left[\mathrm{ClO}_{4}\right]_{2}[2.09(1), 2.11(1) \AA]$ are slightly shorter than those of $\left[\mathrm{Pt}_{2}(\mathrm{dppm})_{2}(\text { quin })_{2}\right]\left[\mathrm{PF}_{6}\right]_{2} \quad[2.13(1)$, $2.12(1) \AA]$, which are in turn shorter than that of $\left[\mathrm{Pt}_{2}-\right.$ $\left.(\mathrm{dppm})_{2}(\mathrm{tmpy}) \mathrm{Cl}\right] \mathrm{PF}_{6}[2.15(1) \AA]$. This may reflect the difference in basicity of the ligands. The Pt-P distances are comparable with those found in other reported $\mathrm{Pt}^{\mathrm{I}}-\mathrm{Pt}^{\mathbf{I}}$ complexes of dppm.

Proton and ${ }^{31} \mathrm{P}$ NMR Spectra.-All the complexes display a pseudo-triplet at around $\delta 4.5-5.5$ for the methylene protons of the dppm ligand with coupling constants $J(\mathrm{P}-\mathrm{H})=10 \mathrm{~Hz}$ and $J(\mathrm{Pt}-\mathrm{H})=50 \mathrm{~Hz}$, characteristic of a bridging dppm ligand. ${ }^{12}$ Complexes with two identical axial ligands display a triplet in their ${ }^{31} \mathrm{P}$ NMR spectra, indicating equivalence of the phosphorus atoms of the dppm ligands in solution. As expected, the complex $\left[\mathrm{Pt}_{2}(\mathrm{dppm})_{2}(\mathrm{tmpy}) \mathrm{Cl}\right] \mathrm{PF}_{6}$, which contains mixed axial ligands, has a ${ }^{31} \mathrm{P}$ NMR spectrum consisting of two triplets. $(\delta 9.03$ and -0.06$)$. The ${ }^{31} \mathrm{P}$ NMR data for these complexes are summarized in Table 4. 
Table 2 Atomic parameters of non-hydrogen atoms with estimated standard deviations (e.s.d.s)

\begin{tabular}{|c|c|c|c|c|c|c|c|}
\hline \multicolumn{8}{|c|}{$\left[\mathrm{Pt}(\mathrm{dppm})_{2}(\mathrm{mim})_{2}\right]\left[\mathrm{ClO}_{4}\right]_{2} \cdot \mathrm{MeCN}$} \\
\hline Atom & $x$ & $y$ & $z$ & Atom & $x$ & $y$ & $z$ \\
\hline $\operatorname{Pt}(1)$ & $0.37719(3)$ & $0.72377(5)$ & $0.06729(5)$ & $\mathrm{C}(24 \mathrm{~B})$ & $0.5407(11)$ & $1.0001(22)$ & $-0.2089(13)$ \\
\hline $\operatorname{Pt}(2)$ & $0.47184(3)$ & $0.74286(6)$ & 0.00034 & $C(25 B)$ & $0.4983(12)$ & $0.9368(19)$ & $-0.2261(12)$ \\
\hline$P(1)$ & $0.32633(20)$ & $0.7248(4)$ & $-0.02761(20)$ & $C(26 B)$ & $0.4586(8)$ & $0.8998(14)$ & $-0.1795(9)$ \\
\hline $\mathrm{P}(2)$ & $0.42248(23)$ & $0.8680(4)$ & $-0.05294(24)$ & $\mathrm{C}(31 \mathrm{~A})$ & $0.4036(7)$ & $0.6703(14)$ & $0.2294(8)$ \\
\hline$P(3)$ & $0.43854(21)$ & $0.6962(4)$ & $0.15116(22)$ & $\mathrm{C}(32 \mathrm{~A})$ & $0.3704(8)$ & $0.7517(16)$ & $0.2574(8)$ \\
\hline$P(4)$ & $0.50614(21)$ & $0.5969(4)$ & $0.04843(20)$ & $\mathrm{C}(33 \mathrm{~A})$ & $0.3444(9)$ & $0.7377(17)$ & $0.3160(9)$ \\
\hline$C(1)$ & $0.3631(8)$ & $0.7997(13)$ & $-0.0911(7)$ & $C(34 A)$ & $0.3534(10)$ & $0.6490(19)$ & $0.3467(9)$ \\
\hline$C(2)$ & $0.4770(7)$ & $0.5813(14)$ & $0.1284(8)$ & $\mathrm{C}(35 \mathrm{~A})$ & $0.3815(13)$ & $0.5704(18)$ & $0.3224(11)$ \\
\hline $\mathrm{N}(11)$ & $0.2998(6)$ & $0.7149(11)$ & $0.1224(6)$ & $\mathrm{C}(36 \mathrm{~A})$ & $0.4082(9)$ & $0.5832(16)$ & $0.2616(9)$ \\
\hline $\mathrm{C}(12)$ & $0.2660(9)$ & $0.7919(13)$ & $0.1374(9)$ & $\mathrm{C}(31 \mathrm{~B})$ & $0.4991(7)$ & $0.7835(14)$ & $0.1740(8)$ \\
\hline $\mathrm{N}(13)$ & $0.2180(6)$ & $0.7613(13)$ & $0.1693(7)$ & $\mathrm{C}(32 \mathrm{~B})$ & $0.5297(8)$ & $0.7647(17)$ & $0.2318(8)$ \\
\hline$C(14)$ & $0.2234(9)$ & $0.6619(17)$ & $0.1794(9)$ & $C(33 B)$ & $0.5792(9)$ & $0.8201(16)$ & $0.2458(9)$ \\
\hline $\mathrm{C}(15)$ & $0.2721(8)$ & $0.6294(14)$ & $0.1486(9)$ & $C(34 B)$ & $0.5971(10)$ & $0.8921(18)$ & $0.2039(10)$ \\
\hline$C(16)$ & $0.1700(9)$ & $0.8286(19)$ & $0.1881(12)$ & $C(35 B)$ & $0.5670(11)$ & $0.9162(17)$ & $0.1515(11)$ \\
\hline$N(21)$ & $0.5499(6)$ & $0.7646(11)$ & $-0.0505(6)$ & $\mathrm{C}(36 \mathrm{~B})$ & $0.5174(10)$ & $0.8644(14)$ & $0.1350(9)$ \\
\hline$C(22)$ & $0.5642(8)$ & $0.7335(16)$ & $-0.1101(9)$ & $\mathrm{C}(41 \mathrm{~A})$ & $0.5856(8)$ & $0.5913(13)$ & $0.0567(8)$ \\
\hline $\mathrm{N}(23)$ & $0.6195(7)$ & $0.7578(14)$ & $-0.1258(8)$ & $\mathrm{C}(42 \mathrm{~A})$ & $0.6130(8)$ & $0.6338(16)$ & $0.1121(8)$ \\
\hline$C(24)$ & $0.6444(8)$ & $0.8049(15)$ & $-0.0736(11)$ & $\mathrm{C}(43 \mathrm{~A})$ & $0.6771(9)$ & $0.6359(18)$ & $0.1106(10)$ \\
\hline$C(25)$ & $0.6017(9)$ & $0.8076(15)$ & $-0.0296(9)$ & $C(44 A)$ & $0.7059(8)$ & $0.6026(15)$ & $0.0593(12)$ \\
\hline $\mathrm{C}(26)$ & $0.6466(12)$ & $0.734(3)$ & $-0.1858(12)$ & $\mathrm{C}(45 \mathrm{~A})$ & $0.6803(8)$ & $0.5646(14)$ & $0.0064(11)$ \\
\hline$C(11 \mathrm{~A})$ & $0.2511(7)$ & $0.7693(13)$ & $-0.0191(7)$ & $\mathrm{C}(46 \mathrm{~A})$ & $0.6192(7)$ & $0.5591(13)$ & $0.0041(9)$ \\
\hline $\mathrm{C}(12 \mathrm{~A})$ & $0.2336(8)$ & $0.8715(15)$ & $-0.0370(9)$ & $C(41 B)$ & $0.4917(7)$ & $0.4813(14)$ & $0.0044(9)$ \\
\hline$C(13 A)$ & $0.1785(9)$ & $0.9055(16)$ & $-0.0237(9)$ & $\mathrm{C}(42 \mathrm{~B})$ & $0.4984(9)$ & $0.3830(14)$ & $0.0328(9)$ \\
\hline$C(14 A)$ & $0.1400(9)$ & $0.8401(16)$ & $0.0047(13)$ & $\mathrm{C}(43 \mathrm{~B})$ & $0.4901(9)$ & $0.2938(13)$ & $-0.0026(13)$ \\
\hline$C(15 A)$ & $0.1541(7)$ & $0.7395(16)$ & $0.0171(9)$ & $C(44 B)$ & $0.4771(9)$ & $0.3017(17)$ & $-0.0692(10)$ \\
\hline$C(16 A)$ & $0.2104(7)$ & $0.7079(13)$ & $0.0053(10)$ & $\mathrm{C}(45 \mathrm{~B})$ & $0.4722(11)$ & $0.3994(17)$ & $-0.0977(9)$ \\
\hline$C(11 B)$ & $0.3200(8)$ & $0.6008(13)$ & $-0.0641(8)$ & $C(46 B)$ & $0.4778(9)$ & $0.4837(15)$ & $-0.0596(8)$ \\
\hline$C(12 B)$ & $0.3331(9)$ & $0.5146(17)$ & $-0.0289(8)$ & $\mathrm{Cl}(1)$ & $0.9456(3)$ & $0.3906(5)$ & $0.7477(3)$ \\
\hline$C(13 B)$ & $0.3269(9)$ & $0.4173(13)$ & $-0.0567(10)$ & $O(11)$ & $0.9081(9)$ & $0.3391(13)$ & $0.7849(8)$ \\
\hline$C(14 B)$ & $0.3030(9)$ & $0.4064(17)$ & $-0.1165(11)$ & $\mathrm{O}(12)$ & $1.0010(9)$ & $0.3726(16)$ & $0.7626(11)$ \\
\hline$C(15 B)$ & $0.2897(11)$ & $0.4923(18)$ & $-0.1541(9)$ & $\mathrm{O}(13)$ & $0.9400(9)$ & $0.3511(17)$ & $0.6890(8)$ \\
\hline$C(16 B)$ & $0.2957(9)$ & $0.5879(14)$ & $-0.1265(8)$ & $O(14)$ & $0.9317(9)$ & $0.4918(16)$ & $0.7428(9)$ \\
\hline$C(21 A)$ & $0.3860(9)$ & $0.9768(13)$ & $-0.0120(8)$ & $\mathrm{Cl}(2)$ & $0.7464(3)$ & $0.9598(5)$ & $0.7876(3)$ \\
\hline $\mathrm{C}(22 \mathrm{~A})$ & $0.3613(10)$ & $1.0515(12)$ & $-0.0500(9)$ & $\mathrm{O}(21)$ & $0.7635(12)$ & $0.8618(15)$ & $0.8073(14)$ \\
\hline$C(23 A)$ & $0.3263(12)$ & $1.1285(15)$ & $-0.0239(11)$ & $\mathrm{O}(22)$ & $0.7397(12)$ & $0.9537(17)$ & $0.7246(9)$ \\
\hline$C(24 A)$ & $0.3154(11)$ & $1.1252(15)$ & $0.0403(10)$ & $O(23)$ & $0.7006(9)$ & $0.9718(17)$ & $0.8162(12)$ \\
\hline $\mathrm{C}(25 \mathrm{~A})$ & $0.3401(11)$ & $1.0520(14)$ & $0.0814(11)$ & $\mathrm{O}(24)$ & $0.7912(9)$ & $1.0276(17)$ & $0.8039(8)$ \\
\hline$C(26 A)$ & $0.3768(11)$ & $0.9809(14)$ & $0.0505(10)$ & $C(51)$ & $0.5863(20)$ & $0.763(3)$ & $-0.3327(20)$ \\
\hline$C(21 B)$ & $0.4653(8)$ & $0.9280(13)$ & $-0.1167(8)$ & $\mathrm{C}(52)$ & $0.6242(21)$ & $0.673(3)$ & $-0.3573(19)$ \\
\hline $\mathrm{C}(22 \mathrm{~B})$ & $0.5082(10)$ & $0.9993(19)$ & $-0.0961(9)$ & $N(53)$ & $0.6459(12)$ & $0.6136(19)$ & $-0.3726(14)$ \\
\hline$C(23 B)$ & $0.5449(10)$ & $1.0353(16)$ & $-0.1440(12)$ & & & & \\
\hline \multicolumn{8}{|c|}{$\left[\mathrm{Pt}_{2}(\mathrm{dppm})_{2}(\text { quin })_{2}\right]\left[\mathrm{PF}_{6}\right]_{2} \cdot 2 \mathrm{CH}_{2} \mathrm{Cl}_{2}$} \\
\hline $\operatorname{Pt}(1)$ & $0.76821(6)$ & $0.32007(5)$ & $0.26757(3)$ & $C(23 B)$ & $0.8633(16)$ & $0.1131(13)$ & $-0.0141(8)$ \\
\hline $\operatorname{Pt}(2)$ & $0.77945(6)$ & $0.15712(5)$ & $0.22915(3)$ & $C(24 B)$ & $0.8093(17)$ & $0.1986(14)$ & $-0.0492(9)$ \\
\hline$P(1)$ & $0.9506(4)$ & $0.3253(3)$ & $0.21813(21)$ & $\mathrm{C}(25 \mathrm{~B})$ & $0.7893(17)$ & $0.2841(14)$ & $-0.0283(9)$ \\
\hline $\mathbf{P}(3)$ & $0.5774(4)$ & $0.3305(3)$ & $0.28749(21)$ & $\mathrm{C}(26 \mathrm{~B})$ & $0.8252(15)$ & $0.2846(12)$ & $0.0300(8)$ \\
\hline$P(2)$ & $0.9142(4)$ & $0.1948(3)$ & $0.14223(20)$ & $C(31 A)$ & $0.4990(16)$ & $0.4157(12)$ & $0.3416(8)$ \\
\hline$P(4)$ & $0.6589(4)$ & $0.1183(3)$ & $0.32239(21)$ & $\mathrm{C}(32 \mathrm{~A})$ & $0.4404(19)$ & $0.5072(15)$ & $0.3165(9)$ \\
\hline$C(1)$ & $0.9553(14)$ & $0.3093(11)$ & $0.1368(7)$ & $C(33 A)$ & $0.3808(21)$ & $0.5702(17)$ & $0.3596(11)$ \\
\hline $\mathrm{C}(2)$ & $0.5314(14)$ & $0.2166(11)$ & $0.3239(7)$ & $C(34 A)$ & $0.3911(20)$ & $0.5376(16)$ & $0.4226(10)$ \\
\hline $\mathrm{N}(1 \mathrm{~A})$ & $0.7544(12)$ & $0.4540(9)$ & $0.2983(6)$ & $C(35 A)$ & $0.4470(21)$ & $0.4515(17)$ & $0.4498(11)$ \\
\hline$C(2 A)$ & $0.7302(15)$ & $0.5382(11)$ & $0.2566(9)$ & $C(36 A)$ & $0.5054(18)$ & $0.3873(14)$ & $0.4059(9)$ \\
\hline$C(3 A)$ & $0.7433(16)$ & $0.6293(14)$ & $0.2748(8)$ & $C(31 B)$ & $0.5173(14)$ & $0.3760(11)$ & $0.2162(7)$ \\
\hline$C(4 A)$ & $0.7824(16)$ & $0.6276(13)$ & $0.3270(9)$ & $\mathrm{C}(32 \mathrm{~B})$ & $0.4049(18)$ & $0.3791(14)$ & $0.2169(9)$ \\
\hline$C(5 A)$ & $0.8052(15)$ & $0.5387(12)$ & $0.3697(8)$ & $C(33 B)$ & $0.3550(18)$ & $0.4072(14)$ & $0.1598(9)$ \\
\hline$C(6 A)$ & $0.8484(19)$ & $0.5310(15)$ & $0.4263(10)$ & $\mathrm{C}(34 \mathrm{~B})$ & $0.4213(16)$ & $0.4343(13)$ & $0.1039(8)$ \\
\hline$C(7 A)$ & $0.8731(21)$ & $0.4469(16)$ & $0.4670(9)$ & $\mathrm{C}(35 \mathrm{~B})$ & $0.5350(17)$ & $0.4297(14)$ & $0.1007(9)$ \\
\hline$C(8 A)$ & $0.8525(17)$ & $0.3613(15)$ & $0.4533(8)$ & $\mathrm{C}(36 \mathrm{~B})$ & $0.5813(15)$ & $0.4011(12)$ & $0.1581(8)$ \\
\hline $\mathrm{C}(9 \mathrm{~A})$ & $0.8132(15)$ & $0.3642(12)$ & $0.3997(8)$ & $\mathrm{C}(41 \mathrm{~A})$ & $0.7096(15)$ & $0.1076(12)$ & $0.3967(8)$ \\
\hline $\mathrm{C}(10 \mathrm{~A})$ & $0.7894(14)$ & $0.4525(12)$ & $0.3550(8)$ & $\mathrm{C}(42 \mathrm{~A})$ & $0.8251(16)$ & $0.0823(13)$ & $0.3974(8)$ \\
\hline$N(1 B)$ & $0.7668(11)$ & $0.0344(9)$ & $0.1942(6)$ & $C(43 A)$ & $0.8643(18)$ & $0.0728(14)$ & $0.4545(9)$ \\
\hline $\mathrm{C}(2 \mathrm{~B})$ & $0.8302(15)$ & $-0.0516(12)$ & $0.2121(8)$ & $\mathrm{C}(44 \mathrm{~A})$ & $0.7924(18)$ & $0.0938(15)$ & $0.5090(9)$ \\
\hline$C(3 B)$ & $0.8267(17)$ & $-0.1380(12)$ & $0.1854(9)$ & $\mathrm{C}(45 \mathrm{~A})$ & $0.6796(20)$ & $0.1131(16)$ & $0.5098(10)$ \\
\hline$C(4 B)$ & $0.7604(17)$ & $-0.1283(13)$ & $0.1424(8)$ & $C(46 A)$ & $0.6356(18)$ & $0.1228(14)$ & $0.4540(9)$ \\
\hline $\mathrm{C}(5 \mathrm{~B})$ & $0.6902(16)$ & $-0.0368(12)$ & $0.1240(8)$ & $\mathrm{C}(41 \mathrm{~B})$ & $0.6083(14)$ & $0.0073(11)$ & $0.3319(7)$ \\
\hline $\mathrm{C}(6 \mathrm{~B})$ & $0.6175(17)$ & $-0.0227(14)$ & $0.0799(8)$ & $\mathrm{C}(42 \mathrm{~B})$ & $0.6441(16)$ & $-0.0745(13)$ & $0.3772(8)$ \\
\hline $\mathrm{C}(7 \mathrm{~B})$ & $0.5528(17)$ & $0.0674(16)$ & $0.0625(9)$ & $\mathrm{C}(43 \mathrm{~B})$ & $0.6060(17)$ & $-0.1620(14)$ & $0.3833(9)$ \\
\hline $\mathrm{C}(8 \mathrm{~B})$ & $0.5563(16)$ & $0.1480(14)$ & $0.0888(8)$ & $\mathrm{C}(44 \mathrm{~B})$ & $0.5376(18)$ & $-0.1654(14)$ & $0.3423(9)$ \\
\hline $\mathrm{C}(9 \mathrm{~B})$ & $0.6285(15)$ & $0.1367(12)$ & $0.1313(8)$ & $\mathrm{C}(45 \mathrm{~B})$ & $0.5012(17)$ & $-0.0887(14)$ & $0.2979(9)$ \\
\hline$C(10 B)$ & $0.6966(14)$ & $0.0435(12)$ & $0.1500(7)$ & $\mathrm{C}(46 \mathrm{~B})$ & $0.5409(15)$ & $0.0010(12)$ & $0.2929(7)$ \\
\hline$C(11 A)$ & $0.9944(13)$ & $0.4389(11)$ & $0.2108(7)$ & $\mathrm{P}(\mathrm{A})$ & $0.0290(5)$ & $0.7953(4)$ & $0.3670(3)$ \\
\hline$C(12 A)$ & $0.9828(15)$ & $0.5117(12)$ & $0.1551(8)$ & $\mathrm{F}(1 \mathrm{~A})$ & $0.1525(17)$ & $0.7930(13)$ & $0.3646(8)$ \\
\hline
\end{tabular}


Table 2 (continued)

\begin{tabular}{|c|c|c|c|c|c|c|c|}
\hline$C(13 A)$ & $1.0147(17)$ & $0.5994(14)$ & $0.1502(9)$ & $F(2 A)$ & $0.0037(13)$ & $0.9051(10)$ & $0.3388(6)$ \\
\hline$C(14 A)$ & $1.0567(17)$ & $0.6197(13)$ & $0.2013(8)$ & $F(3 A)$ & $0.0080(12)$ & $0.8208(10)$ & $0.4364(6)$ \\
\hline$C(15 A)$ & $1.0687(16)$ & $0.5491(13)$ & $0.2567(8)$ & $F(4 A)$ & $-0.0975(17)$ & $0.7937(14)$ & $0.3750(9)$ \\
\hline$C(16 A)$ & $1.0359(16)$ & $0.4567(13)$ & $0.2577(8)$ & $F(5 A)$ & $0.0496(15)$ & $0.6822(12)$ & $0.3950(8)$ \\
\hline$C(11 B)$ & $1.0681(14)$ & $0.2379(11)$ & $0.2522(7)$ & $F(6 A)$ & $0.0537(13)$ & $0.7766(10)$ & $0.2956(7)$ \\
\hline $\mathrm{C}(12 \mathrm{~B})$ & $1.0487(15)$ & $0.1858(12)$ & $0.3117(8)$ & $P(B)$ & $0.7497(6)$ & $0.6403(5)$ & $0.0243(3)$ \\
\hline$C(13 B)$ & $1.1370(18)$ & $0.1186(14)$ & $0.3393(9)$ & $F(1 B)$ & $0.7658(12)$ & $0.6692(10)$ & $-0.0513(6)$ \\
\hline$C(14 B)$ & $1.1760(16)$ & $0.2244(13)$ & $0.2120(8)$ & $F(2 B)$ & $0.8727(14)$ & $0.6505(11)$ & $0.0209(7)$ \\
\hline$C(15 B)$ & $1.2623(19)$ & $0.1607(15)$ & $0.2456(9)$ & $F(3 B)$ & $0.7077(14)$ & $0.7486(12)$ & $0.0255(7)$ \\
\hline$C(16 B)$ & $1.2424(17)$ & $0.1081(14)$ & $0.3043(9)$ & $F(4 B)$ & $0.7327(15)$ & $0.6119(12)$ & $0.1000(7)$ \\
\hline$C(21 A)$ & $1.0458(14)$ & $0.1055(11)$ & $0.1418(7)$ & $F(5 B)$ & $0.6220(13)$ & $0.6349(10)$ & $0.0265(6)$ \\
\hline$C(22 A)$ & $1.0686(15)$ & $0.0326(12)$ & $0.1939(7)$ & $F(6 B)$ & $0.7849(16)$ & $0.5358(13)$ & $0.0214(8)$ \\
\hline$C(23 A)$ & $1.1726(16)$ & $-0.0334(13)$ & $0.1941(8)$ & $\mathrm{Cl}(1)$ & $0.5032(8)$ & $0.7574(6)$ & $0.1869(4)$ \\
\hline$C(24 A)$ & $1.2570(17)$ & $-0.0220(14)$ & $0.1432(9)$ & $\mathrm{C}(\mathrm{A})$ & $0.452(3)$ & $0.6938(23)$ & $0.1416(14)$ \\
\hline$C(25 A)$ & $1.2366(17)$ & $0.0487(13)$ & $0.0921(9)$ & $\mathrm{Cl}(2)$ & $0.3218(8)$ & $0.6744(6)$ & $0.1857(4)$ \\
\hline$C(26 A)$ & $1.1329(15)$ & $0.1149(12)$ & $0.0894(8)$ & $\mathrm{Cl}(3)$ & $0.2017(8)$ & $0.3675(7)$ & $0.4016(4)$ \\
\hline $\mathrm{C}(21 \mathrm{~B})$ & $0.8786(14)$ & $0.1998(11)$ & $0.0645(7)$ & $\mathrm{C}(\mathrm{B})$ & $0.185(3)$ & $0.2661(20)$ & $0.4510(13)$ \\
\hline$C(22 B)$ & $0.8965(15)$ & $0.1128(12)$ & $0.0423(8)$ & $\mathrm{Cl}(4)$ & $0.2889(7)$ & $0.1684(6)$ & $0.4578(4)$ \\
\hline \multicolumn{8}{|c|}{$\left[\mathrm{Pt}_{2}(\mathrm{dppm})_{2}(\mathrm{tmpy}) \mathrm{Cl}\right] \mathrm{PF}_{6} \cdot \mathrm{Me}_{2} \mathrm{CO}$} \\
\hline $\operatorname{Pt}(1)$ & $0.34121(5)$ & $0.37242(5)$ & $0.21593(3)$ & $\mathrm{C}(23 \mathrm{~B})$ & $0.2742(12)$ & $0.4284(11)$ & $0.0118(8)$ \\
\hline $\operatorname{Pt}(2)$ & $0.39626(5)$ & $0.23029(5)$ & $0.17917(3)$ & $\mathrm{C}(24 \mathrm{~B})$ & $0.1996(13)$ & $0.4254(12)$ & $-0.0227(9)$ \\
\hline$P(1)$ & $0.2110(3)$ & $0.3207(3)$ & $0.20332(22)$ & $\mathrm{C}(25 \mathrm{~B})$ & $0.1466(13)$ & $0.3609(13)$ & $-0.0176(9)$ \\
\hline $\mathbf{P}(2)$ & $0.2766(3)$ & $0.2196(3)$ & $0.11439(21)$ & $C(26 B)$ & $0.1681(12)$ & $0.2989(11)$ & $0.0236(8)$ \\
\hline$P(3)$ & $0.4716(3)$ & $0.4237(3)$ & $0.22587(23)$ & $C(31 A)$ & $0.5027(11)$ & $0.5012(12)$ & $0.2871(8)$ \\
\hline$P(4)$ & $0.5025(3)$ & $0.2483(3)$ & $0.25559(22)$ & $C(32 A)$ & $0.4718(12)$ & $0.4928(12)$ & $0.3398(8)$ \\
\hline$P(5)$ & $0.7390(4)$ & $0.2954(4)$ & $0.0708(3)$ & $C(33 A)$ & $0.4956(14)$ & $0.5485(14)$ & $0.3880(9)$ \\
\hline $\mathrm{Cl}$ & $0.2953(3)$ & $0.5075(3)$ & $0.24584(24)$ & $C(34 A)$ & $0.5503(14)$ & $0.6102(14)$ & $0.3815(9)$ \\
\hline $\mathrm{N}$ & $0.4514(10)$ & $0.1255(9)$ & $0.1413(7)$ & $C(35 A)$ & $0.5805(14)$ & $0.6185(13)$ & $0.3306(10)$ \\
\hline$C(1)$ & $0.1987(11)$ & $0.2203(10)$ & $0.1597(7)$ & $C(36 A)$ & $0.5594(12)$ & $0.5667(12)$ & $0.2826(9)$ \\
\hline$C(2)$ & $0.5539(11)$ & $0.3439(11)$ & $0.2419(8)$ & $C(31 B)$ & $0.4943(12)$ & $0.4773(12)$ & $0.1576(8)$ \\
\hline$C(3)$ & $0.4900(12)$ & $0.1329(15)$ & $0.0955(9)$ & $C(32 B)$ & $0.5731(13)$ & $0.4806(14)$ & $0.1431(9)$ \\
\hline$C(4)$ & $0.5300(14)$ & $0.0644(13)$ & $0.0741(9)$ & $C(33 B)$ & $0.5860(17)$ & $0.5235(15)$ & $0.0933(9)$ \\
\hline$C(5)$ & $0.5308(14)$ & $-0.0121(16)$ & $0.1015(10)$ & $C(34 B)$ & $0.5241(17)$ & $0.5660(14)$ & $0.0594(10)$ \\
\hline$C(6)$ & $0.4895(14)$ & $-0.0222(12)$ & $0.1480(12)$ & $C(35 B)$ & $0.4476(16)$ & $0.5639(14)$ & $0.0715(10)$ \\
\hline$C(7)$ & $0.4500(11)$ & $0.0478(12)$ & $0.1697(9)$ & $C(36 B)$ & $0.4331(12)$ & $0.5238(11)$ & $0.1216(9)$ \\
\hline $\mathrm{C}(8)$ & $0.4878(15)$ & $0.2159(12)$ & $0.0658(10)$ & $C(41 A)$ & $0.4780(12)$ & $0.2596(12)$ & $0.3303(8)$ \\
\hline C(9) & $0.5680(17)$ & $-0.0949(17)$ & $0.0824(12)$ & $C(42 A)$ & $0.5307(15)$ & $0.2942(14)$ & $0.3757(9)$ \\
\hline $\mathrm{C}(10)$ & $0.4100(14)$ & $0.0375(13)$ & $0.2250(10)$ & $\mathrm{C}(43 \mathrm{~A})$ & $0.5134(17)$ & $0.3090(15)$ & $0.4330(10)$ \\
\hline$C(11 A)$ & $0.1748(11)$ & $0.2989(11)$ & $0.2719(9)$ & $C(44 A)$ & $0.4392(16)$ & $0.2799(16)$ & $0.4430(9)$ \\
\hline $\mathrm{C}(12 \mathrm{~A})$ & $0.1096(14)$ & $0.2384(14)$ & $0.2721(9)$ & $C(45 A)$ & $0.3818(16)$ & $0.2412(15)$ & $0.4011(10)$ \\
\hline$C(13 A)$ & $0.0789(15)$ & $0.2285(13)$ & $0.3294(11)$ & $\mathrm{C}(46 \mathrm{~A})$ & $0.4044(14)$ & $0.2341(13)$ & $0.3419(9)$ \\
\hline$C(14 A)$ & $0.1153(15)$ & $0.2696(15)$ & $0.3808(9)$ & $C(41 B)$ & $0.5855(11)$ & $0.1685(11)$ & $0.2612(8)$ \\
\hline$C(15 A)$ & $0.1726(16)$ & $0.3266(13)$ & $0.3781(9)$ & $C(42 B)$ & $0.6344(12)$ & $0.1667(13)$ & $0.2192(9)$ \\
\hline$C(16 A)$ & $0.2034(14)$ & $0.3383(11)$ & $0.3231(9)$ & $C(43 B)$ & $0.6877(12)$ & $0.1022(14)$ & $0.2183(9)$ \\
\hline$C(11 B)$ & $0.1327(10)$ & $0.3812(11)$ & $0.1572(7)$ & $C(44 B)$ & $0.6931(12)$ & $0.0396(13)$ & $0.2623(9)$ \\
\hline$C(12 B)$ & $0.1501(12)$ & $0.4562(12)$ & $0.1295(9)$ & $\mathrm{C}(45 \mathrm{~B})$ & $0.6448(14)$ & $0.0419(12)$ & $0.3049(10)$ \\
\hline$C(13 B)$ & $0.0895(14)$ & $0.4984(13)$ & $0.0921(9)$ & $C(46 B)$ & $0.5896(12)$ & $0.1062(12)$ & $0.3047(8)$ \\
\hline$C(14 B)$ & $0.0141(14)$ & $0.4704(14)$ & $0.0817(11)$ & $\mathrm{O}$ & $0.7643(11)$ & $0.8483(12)$ & $0.1746(10)$ \\
\hline$C(15 B)$ & $-0.0094(13)$ & $0.4027(16)$ & $0.1078(11)$ & $\mathrm{C}(11)$ & $0.7138(15)$ & $0.7940(15)$ & $0.1773(13)$ \\
\hline$C(16 B)$ & $0.0507(12)$ & $0.3594(13)$ & $0.1477(9)$ & $C(12)$ & $0.6644(21)$ & $0.7911(20)$ & $0.2244(13)$ \\
\hline$C(21 A)$ & $0.2540(11)$ & $0.1250(11)$ & $0.0691(7)$ & $C(13)$ & $0.7053(22)$ & $0.7275(17)$ & $0.1376(12)$ \\
\hline$C(22 A)$ & $0.2652(14)$ & $0.1217(13)$ & $0.0108(9)$ & $F(1)$ & $0.6851(14)$ & $0.3090(13)$ & $0.1145(10)$ \\
\hline$C(23 A)$ & $0.2439(18)$ & $0.0496(15)$ & $-0.0236(11)$ & $F(2)$ & $0.8170(12)$ & $0.2808(15)$ & $0.1070(12)$ \\
\hline$C(24 A)$ & $0.2108(15)$ & $-0.0163(13)$ & $0.0007(10)$ & $F(3)$ & $0.7648(12)$ & $0.3791(11)$ & $0.0748(13)$ \\
\hline$C(25 A)$ & $0.1986(15)$ & $-0.0183(13)$ & $0.0582(10)$ & $F(4)$ & $0.6676(13)$ & $0.3055(19)$ & $0.0291(9)$ \\
\hline$C(26 A)$ & $0.2202(13)$ & $0.0547(12)$ & $0.0934(8)$ & $F(5)$ & $0.7184(15)$ & $0.2138(12)$ & $0.0882(14)$ \\
\hline$C(21 B)$ & $0.2456(12)$ & $0.2999(10)$ & $0.0602(7)$ & $F(6)$ & $0.7839(16)$ & $0.2705(20)$ & $0.0273(10)$ \\
\hline$C(22 B)$ & $0.3005(11)$ & $0.3649(12)$ & $0.0537(8)$ & & & & \\
\hline
\end{tabular}

Electronic Spectroscopy.-.The colour of $\left[\mathrm{Pt}_{2}(\mathrm{dppm})_{2}-\right.$ $\mathrm{X}(\mathrm{Y})]^{n+}$ is dependent on the nature of the axial ligands. The complexes $\left[\mathrm{Pt}_{2}(\mathrm{dppm})_{2}\left(\mathrm{PPh}_{3}\right)_{2}\right]^{2+}$ and $\left[\mathrm{Pt}_{2}(\mathrm{dppm})_{2}(\mathrm{tmpy})-\right.$ $\mathrm{Cl}]^{2+}$ are bright yellow whereas $\left[\mathrm{Pt}_{2}(\mathrm{dppm})_{2} \mathrm{~L}_{2}\right]^{2+}(\mathrm{L}=$ nitrogen base) are pale yellow or colourless. First, it is important to locate the ${ }^{1}\left(d_{\sigma} \longrightarrow d_{\sigma^{*}}\right)$ transition $\left(d_{\sigma}\right.$ and $d_{\sigma^{*}}$ refer to the bonding and antibonding combinations of $d_{z^{2}}$ orbitals) which is commonly observed for $d^{7}-d^{7}$ complexes. The energy of the transition is determined by the extent of $d_{\sigma}-d_{\sigma^{*}}$ splitting and hence the extent of intramolecular metal-metal interaction. The metal-metal separation, the nature of axially co-ordinated ligands and the formal charge on the metal ions are factors affecting the extent of such interaction. From X-ray crystallographic studies it is known that the intramolecular $\mathrm{Pt}-\mathrm{Pt}$ distances in $\left[\mathrm{Pt}_{2}(\mathrm{dppm})_{2} \mathrm{X}(\mathrm{Y})\right]^{n+}$ are longer than those found in $\left[\mathrm{Pt}^{\mathrm{II}}{ }_{2}\left(\mathrm{HPO}_{4}\right)_{4} \mathrm{~L}_{2}\right]^{n-}$ or $\left[\mathrm{Pt}^{\mathrm{III}}{ }_{2}\left(\mathrm{SO}_{4}\right)_{4} \mathrm{~L}_{2}\right]^{n-}(2.461-$ $2.529 \AA)^{13}$ but shorter than that in $\left[\mathrm{Pt}^{\mathrm{III}}{ }_{2}\left(\mathrm{H}_{2} \mathrm{P}_{2} \mathrm{O}_{5}\right)_{4} \mathrm{~L}_{2}\right]^{n-}(n=$ 2 or 4$){ }^{3 a, b}$ For all these $\mathrm{Pt}^{\mathrm{III}}-\mathrm{Pt} \mathrm{III}^{\mathrm{II}}$ complexes the ${ }^{1}\left(\mathrm{~d}_{\sigma} \longrightarrow \mathrm{d}_{\sigma^{*}}\right)$ transitions are found in the region $200-300 \mathrm{~nm} .^{7,14}$ The benchmark of the transition found for the complex $\left[\mathrm{Pt}_{2}\left(\mathrm{H}_{2} \mathrm{P}_{2} \mathrm{O}_{5}\right)_{4}\right.$ $\left.(\mathrm{MeCN})_{2}\right]^{2-}(\mathrm{Pt}-\mathrm{Pt} 2.676 \AA)$ is located at $215 \mathrm{~nm}{ }^{3 b}$ Based on the metal-metal separation alone, it is expected that the ${ }^{1}\left(\mathrm{~d}_{\sigma} \longrightarrow \mathrm{d}_{\sigma^{*}}\right)$ transition of $\mathbf{P t}^{\mathrm{I}}-\mathbf{P t}^{1}$ complexes may be found in the high-UV region $(<215 \mathrm{~nm})$. However, the effect of the formal charge on the metal ion should not be neglected. Given the fact that both systems contain a metal-metal single bond, 


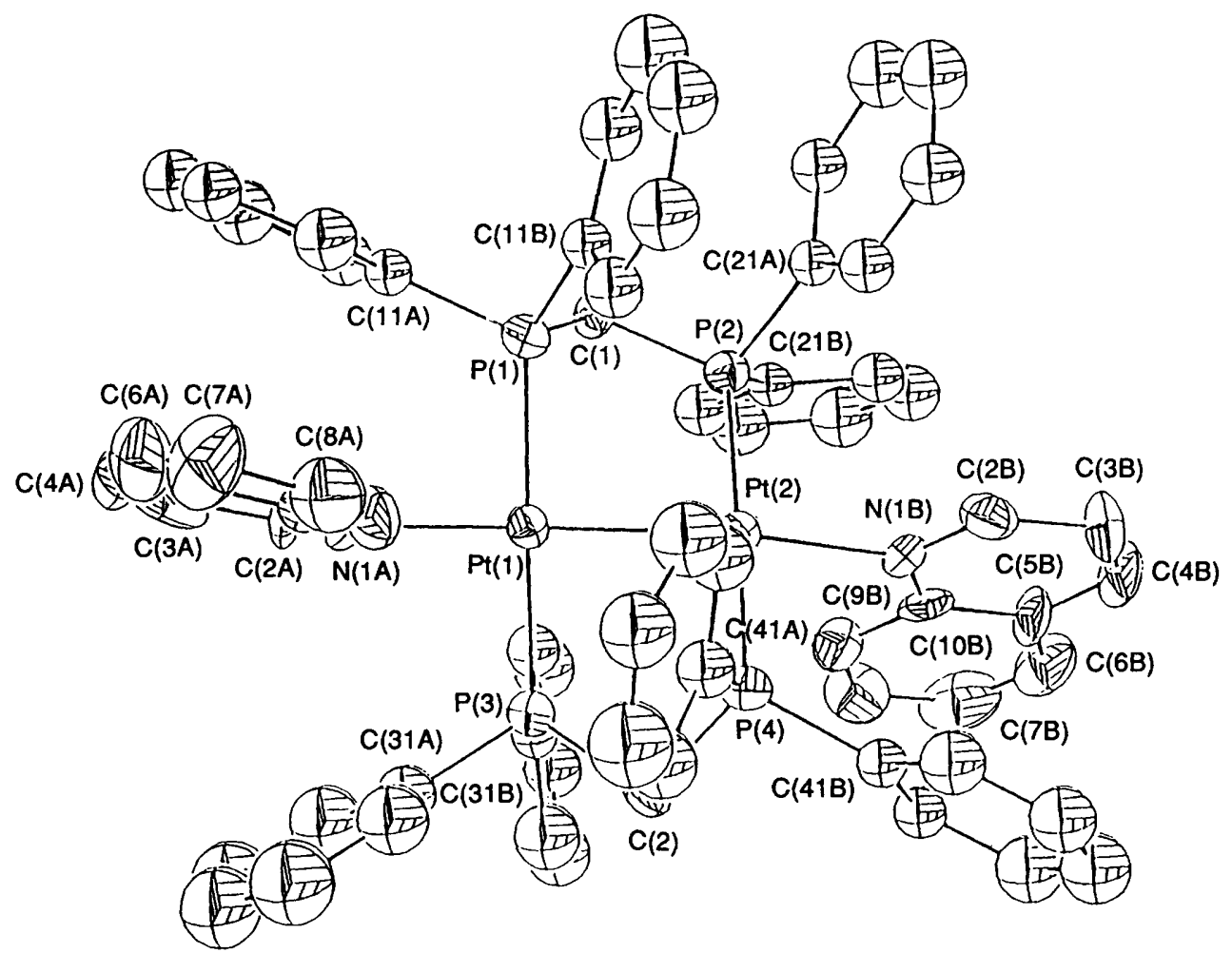

Fig. 1 A perspective view of $\left.\left[\mathrm{Pt}_{2}(\mathrm{dppm})_{2} \text { (quin }\right)_{2}\right]^{2+}$ with atom numbering

Table 3 Selected bond distances $(\AA)$ and angles $\left(^{\circ}\right)$

$\left[\mathrm{Pt}_{2}(\mathrm{dppm})_{2}(\text { quin })_{2}\right]\left[\mathrm{PF}_{6}\right]_{2} \cdot 2 \mathrm{CH}_{2} \mathrm{Cl}_{2}$

$\begin{array}{lllr}\mathrm{Pt}(1)-\mathrm{Pt}(2) & 2.615(1) & \mathrm{Pt}(2)-\mathrm{Pt}(1)-\mathrm{P}(1) & 91.2(1) \\ \mathrm{Pt}(1)-\mathrm{P}(1) & 2.297(5) & \mathrm{Pt}(2)-\mathrm{Pt}(1)-\mathrm{N}(1 \mathrm{~A}) & 178.4(4) \\ \mathrm{Pt}(1)-\mathrm{P}(3) & 2.285(5) & \mathrm{P}(1)-\mathrm{Pt}(1)-\mathrm{P}(3) & 163.7(2) \\ \mathrm{Pt}(1)-\mathrm{N}(1 \mathrm{~A}) & 2.13(1) & \mathrm{P}(1)-\mathrm{Pt}(1)-\mathrm{N}(1 \mathrm{~A}) & 90.0(4) \\ \mathrm{Pt}(2)-\mathrm{P}(2) & 2.293(5) & \mathrm{P}(2)-\mathrm{Pt}(2)-\mathrm{P}(4) & 174.0(2) \\ \mathrm{Pt}(2)-\mathrm{P}(4) & 2.285(5) & \mathrm{P}(2)-\mathrm{Pt}(2)-\mathrm{N}(1 \mathrm{~B}) & 90.7(4) \\ \mathrm{Pt}(2)-\mathrm{N}(1 \mathrm{~B}) & 2.12(1) & \mathrm{Pt}(1)-\mathrm{Pt}(2)-\mathrm{N}(1 \mathrm{~B}) & 172.1(4)\end{array}$

$\left[\mathrm{Pt}_{2}(\mathrm{dppm})_{2}(\mathrm{tmpy}) \mathrm{Cl}\right] \mathrm{PF}_{6} \cdot \mathrm{Me}_{2} \mathrm{CO}$

$\begin{array}{lllr}\mathrm{Pt}(1)-\mathrm{Pt}(2) & 2.627(2) & \mathrm{Pt}(2)-\mathrm{Pt}(1)-\mathrm{P}(1) & 91.1(1) \\ \mathrm{Pt}(1)-\mathrm{P}(1) & 2.281(5) & \mathrm{Pt}(2)-\mathrm{Pt}(1)-\mathrm{P}(3) & 88.2(1) \\ \mathrm{Pt}(1)-\mathrm{P}(3) & 2.284(5) & \mathrm{Pt}(2)-\mathrm{Pt}(1)-\mathrm{Cl} & 176.6(1) \\ \mathrm{Pt}(1)-\mathrm{C}(1) & 2.416(5) & \mathrm{P}(1)-\mathrm{Pt}(1)-\mathrm{P}(3) & 178.5(2) \\ \mathrm{Pt}(2)-\mathrm{P}(2) & 2.267(5) & \mathrm{P}(4)-\mathrm{Pt}(2)-\mathrm{P}(2) & 170.2(2) \\ \mathrm{Pt}(2)-\mathrm{P}(4) & 2.272(5) & \mathrm{Pt}(1)-\mathrm{Pt}(2)-\mathrm{N} & 171.4(4) \\ \mathrm{Pt}(2)-\mathrm{N} & 2.15(1) & \mathrm{P}(2)-\mathrm{Pt}(2)-\mathrm{N} & 93.8(4)\end{array}$

$\left[\mathrm{Pt}_{2}(\mathrm{dppm})_{2}(\mathrm{mim})_{2}\right]\left[\mathrm{ClO}_{4}\right]_{2} \cdot \mathrm{MeCN}$

\begin{tabular}{llll}
$\mathrm{Pt}(1)-\mathrm{Pt}(2)$ & $2.580(1)$ & $\mathrm{Pt}(2)-\mathrm{Pt}(1)-\mathrm{P}(1)$ & $88.1(1)$ \\
$\mathrm{Pt}(1)-\mathrm{P}(1)$ & $2.279(4)$ & $\mathrm{Pt}(2)-\mathrm{Pt}(1)-\mathrm{P}(3)$ & $84.58(3)$ \\
$\mathrm{Pt}(1)-\mathrm{P}(3)$ & $2.257(5)$ & $\mathrm{Pt}(2)-\mathrm{Pt}(1)-\mathrm{N}(11)$ & $177.6(4)$ \\
$\mathrm{Pt}(1)-\mathrm{N}(11)$ & $2.11(1)$ & $\mathrm{P}(1)-\mathrm{Pt}(1)-\mathrm{P}(3)$ & $167.9(2)$ \\
$\mathrm{Pt}(2)-\mathrm{P}(2)$ & $2.269(5)$ & $\mathrm{P}(2)-\mathrm{Pt}(2)-\mathrm{N}(21)$ & $168.7(2)$ \\
$\mathrm{Pt}(2)-\mathrm{P}(4)$ & $2.285(5)$ & $\mathrm{Pt}(1)-\mathrm{Pt}(2)-\mathrm{N}(21)$ & $176.9(4)$ \\
$\mathrm{Pt}(2)-\mathrm{N}(21)$ & $2.09(1)$ & $\mathrm{Pt}(1)-\mathrm{Pt}(2)-\mathrm{P}(2)$ & $85.0(1)$ \\
\hline
\end{tabular}

the $\mathrm{Pt}^{\mathrm{I}}-\mathrm{Pt}^{1}$ interaction is expected to be stronger than $\mathrm{Pt}^{\mathrm{III}}-\mathrm{Pt}^{\mathrm{III}}$ because of the low valency of $\mathrm{Pt}^{\mathrm{I}}$. The cationic nature of $\left[\mathrm{Pt}_{2}(\mathrm{dppm})_{2} \mathrm{X}(\mathrm{Y})\right]^{n+}$ may diminish the metal-metal interaction to some extent but as a whole one would expect that $\left[\mathrm{Pt}_{2}(\mathrm{dppm})_{2} \mathrm{X}(\mathrm{Y})\right]^{n+}$ would have comparable metal-metal interaction to that in $\left[\mathrm{Pt}^{\mathrm{III}}{ }_{2}\left(\mathrm{H}_{2} \mathrm{P}_{2} \mathrm{O}_{5}\right)_{4} \mathrm{~L}_{2}\right]^{n-}(n=2$ or 4$)$. This is further supported by the similar metal-metal distances found in these two classes of complexes. It is therefore reasonable to search for the ${ }^{1}\left(\mathrm{~d}_{\sigma} \longrightarrow \mathrm{d}_{\sigma^{*}}\right)$ transition of $\left[\mathrm{Pt}_{2}(\mathrm{dppm})_{2} \mathrm{X}(\mathrm{Y})\right]^{n+}$
Table 4 Phosphorus-31 NMR data ${ }^{a}$ for the $\mathrm{Pt}^{1}-\mathrm{Pt}^{\mathrm{I}}$ complexes

$\begin{array}{lcl}\text { Complex } & \delta & { }^{1} J(\mathrm{Pt}-\mathrm{P}) / \mathrm{Hz} \\ {\left[\mathrm{Pt}_{2}(\mathrm{dppm})_{2}\left(\mathrm{PPh}_{3}\right)_{2}\right]^{2+}} & -8.27^{b} & 2800 \\ & 14.15 & 1680 \\ {\left[\mathrm{Pt}_{2}(\mathrm{dppm})_{2}(\mathrm{py})_{2}\right]^{2+}} & 0.90^{b} & 2828 \\ {\left[\mathrm{Pt}_{2}(\mathrm{dppm})_{2}(\mathrm{quin})_{2}\right]^{2+}} & 5.67^{c} & 2845 \\ {\left[\mathrm{Pt}_{2}(\mathrm{dppm})_{2}(\mathrm{mim})_{2}\right]^{2+}} & 5.01^{c} & 2745 \\ {\left[\mathrm{Pt}_{2}(\mathrm{dppm})_{2}(\mathrm{tmpy}) \mathrm{Cl}\right]^{+}} & 9.03^{c} & 2885 \\ & -0.06^{c} & 2830 \\ {\left[\mathrm{Pt}_{2}(\mathrm{dppm})_{2}(\mathbf{B B u}-\mathrm{py})_{2}\right]^{2+}} & 5.50^{c} & 2760\end{array}$

a All measurements were made with $\left(\mathrm{CD}_{3}\right)_{2} \mathrm{CO}$ solutions of the hexafluorophosphate salts of the complexes. ${ }^{b}$ Ref. 10 ; trimethyl phosphate was used as reference. ${ }^{c}$ This work; $85 \%$ phosphoric acid was used as reference.

in the high-UV region. Unfortunately, the presence of intense intraligand absorption around $200-300 \mathrm{~nm}$ obscures this region. Attempts to synthesise corresponding $d^{9}-d^{9}$ complexes with bridging ligands like dmpm are now being undertaken in order to locate the transition.

Fig. 4 displays the room-temperature UV/VIS absorption spectrum of an acetonitrile solution of $\left[\mathrm{Pt}_{2}(\mathrm{dppm})_{2}\left(\mathrm{PPh}_{3}\right)_{2}\right]-$ $\left[\mathrm{PF}_{6}\right]_{2}$. There are two intense bands in the visible region (band I, $\lambda_{\max }=350, \varepsilon_{\max }=1.94 \times 10^{4}$; II, $\lambda_{\max }=403 \mathrm{~nm}, \varepsilon_{\max }=$ $\left.1.37 \times 10^{4} \mathrm{dm}^{3} \mathrm{~mol}^{-1} \mathrm{~cm}^{-1}\right)$. We expect that the molecular orbital arrangement in $\mathrm{Pt}^{\mathrm{I}}-\mathrm{Pt}^{\mathrm{I}}$ would be highly analogous to that in $\mathrm{Pt}^{\mathrm{III}}-\mathrm{Pt}^{\mathrm{III}}$ or $\mathrm{Rh}^{\mathrm{II}}-\mathrm{Rh}^{\mathrm{II}}$ complexes with the energy levels in the order: $\mathrm{d}_{\sigma}<\mathrm{d}_{\pi^{\prime}}, \mathrm{d}_{\pi^{*}}, \mathrm{~d}_{\delta}, \mathrm{d}_{\delta^{*}}<\mathrm{d}_{\sigma^{*}}$. Fig. 5 gives a qualitative molecular orbital energy-level diagram. Suitable candidates for the two low-energy transitions are ${ }^{1}\left(\mathrm{~d}_{n^{*}} \longrightarrow \mathrm{d}_{\sigma^{*}}\right)\left({ }^{1} \mathrm{~A}_{\mathrm{g}} \longrightarrow\right.$ $\left.{ }^{1} \mathrm{~B}_{3 \mathrm{u}}\right)$ (symmetry allowed), ${ }^{1}\left(\mathrm{~d}_{\delta^{*}} \longrightarrow \mathrm{d}_{\left.\mathrm{\sigma}^{*}\right)}\left({ }^{1} \mathrm{~A}_{\mathrm{g}} \longrightarrow{ }^{\circ} \longrightarrow{ }^{1} \mathrm{~A}_{\mathrm{g}}\right)\right.$
(symmetry forbidden) and ${ }^{1}\left(\mathrm{~d}_{\delta} \longrightarrow \mathrm{d}_{\sigma^{*}}\right)\left({ }^{1} \mathrm{~A}_{\mathrm{g}} \longrightarrow{ }^{1} \mathrm{~B}_{1 \mathrm{u}}\right)$ (symmetry allowed) (assuming $D_{2 h}$ symmetry in solution). It is reasonable to assign band II to ${ }^{1}\left(\mathrm{~d}_{\pi} \longrightarrow \mathrm{d}_{\sigma^{*}}\right)$ and band I to ${ }^{1}\left(d_{\delta} / d_{\delta^{*}} \longrightarrow d_{\sigma^{*}}\right)$ transitions for the $d_{\pi}-d_{\pi^{*}}$ splitting is expected to be much greater than the $d_{\delta}-d_{\delta^{*}}$. Hence the highest occupied 


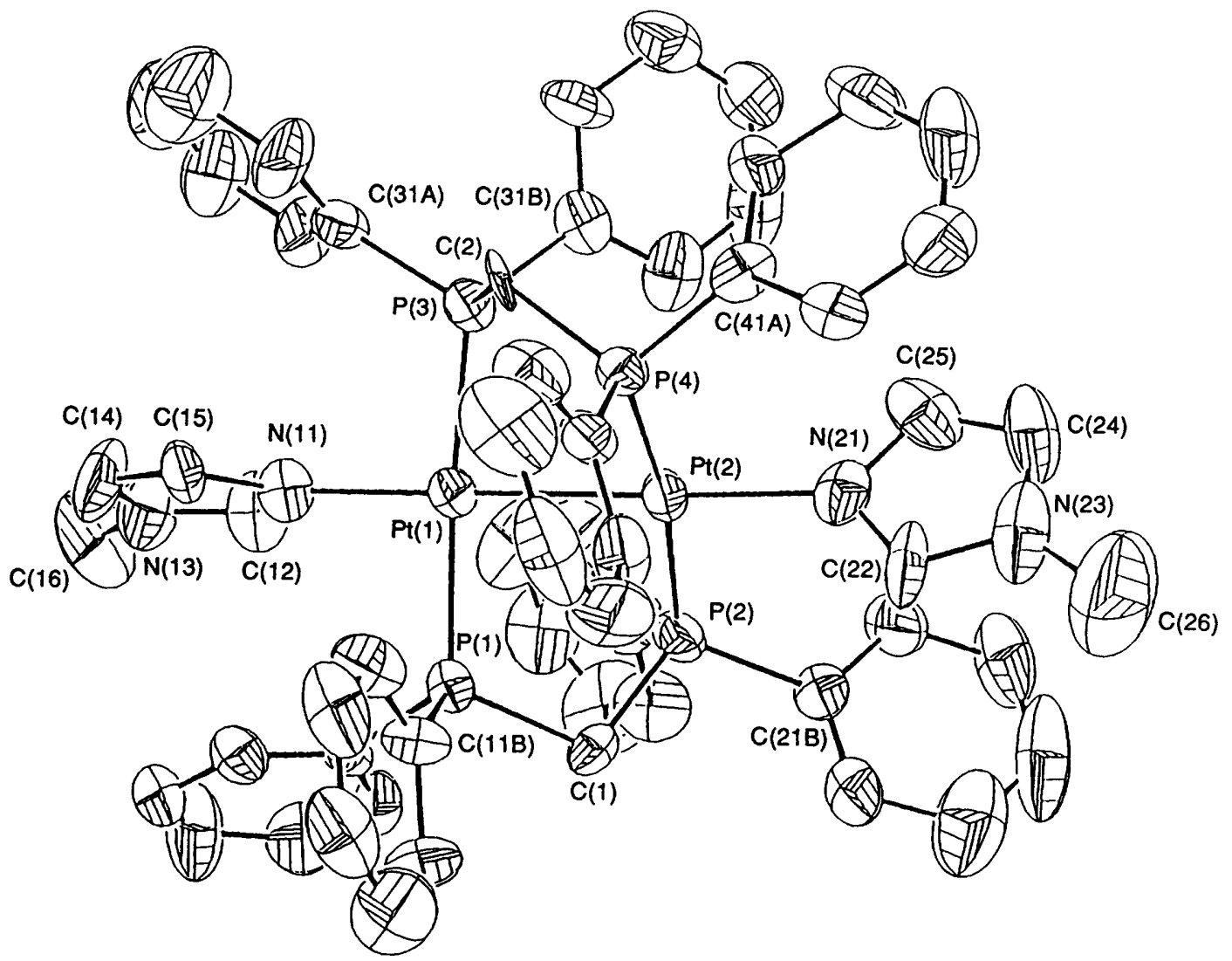

Fig. 2 A perspective view of $\left[\mathrm{Pt}_{2}(\mathrm{dppm})_{2}(\mathrm{mim})_{2}\right]^{2+}$ with atom numbering

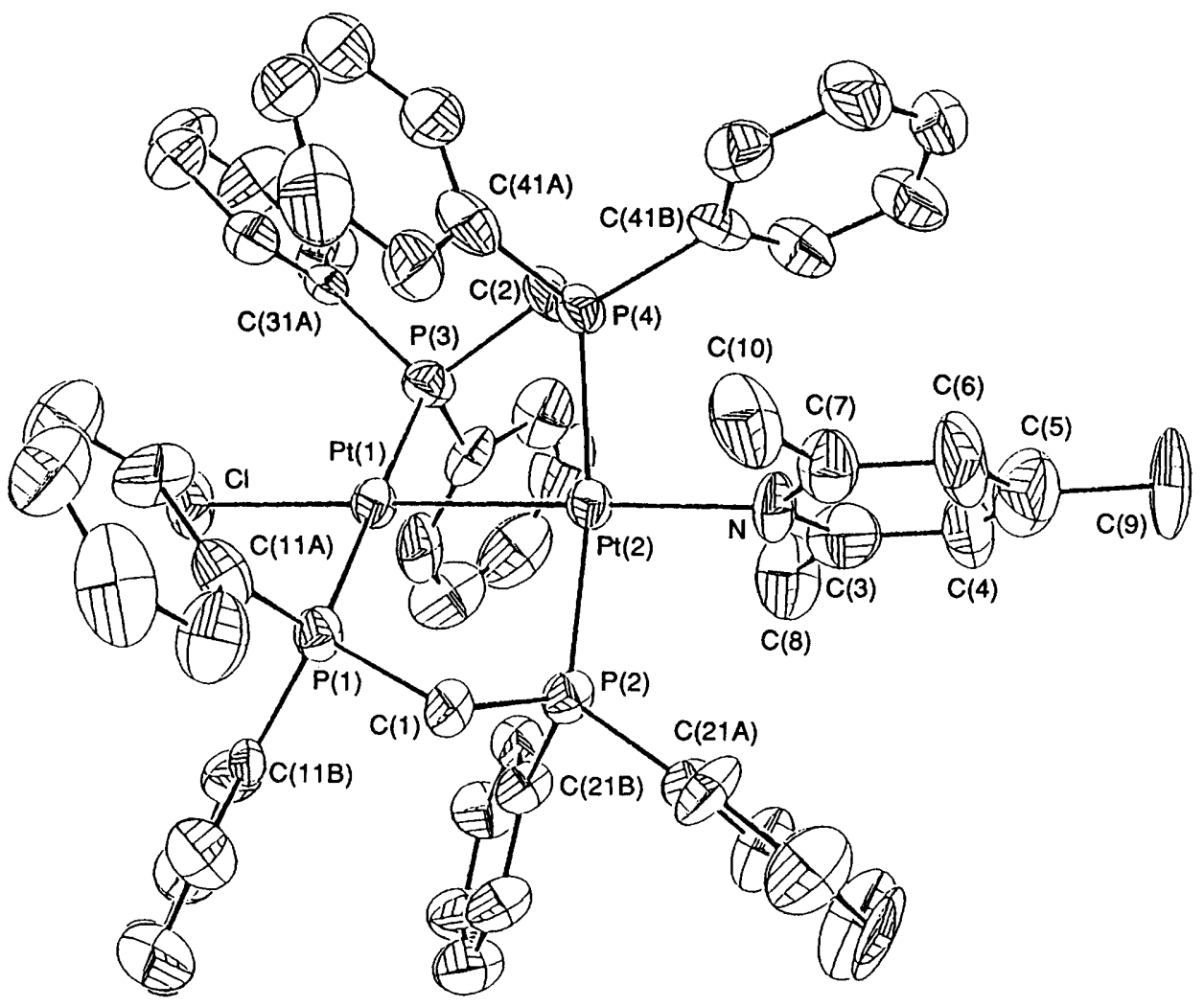

Fig. 3 A perspective view of $\left[\mathrm{Pt}_{2}(\mathrm{dppm})_{2}(\mathrm{tmpy}) \mathrm{Cl}\right]^{+}$with atom numbering

molecular orbital (HOMO) should be the $d_{\pi^{*}}$ orbital. However, the $3760 \mathrm{~cm}^{-1}$ energy separation between the ${ }^{1}\left(d_{x^{*}} \longrightarrow d_{\sigma^{*}}\right)$ and ${ }^{1}\left(d_{\delta} \longrightarrow d_{\sigma^{*}}\right)$ transitions is significantly greater than those found in the $\left[\mathrm{Pt}_{2}\left(\mathrm{HPO}_{4}\right)_{4} \mathrm{Cl}_{2}\right]^{4-}\left(2300 \mathrm{~cm}^{-1}\right)^{7}$ and $\left[\mathrm{Pt}_{2}\left(\mathrm{SO}_{4}\right)_{4}^{-}\right.$ $\left.\left(\mathrm{H}_{2} \mathrm{O}\right)_{2}\right]^{2-}\left(2422 \mathrm{~cm}^{-1}\right){ }^{14}$ This could be explained by the fact that in the two diplatinum(III) complexes the $\delta$ orbitals are 


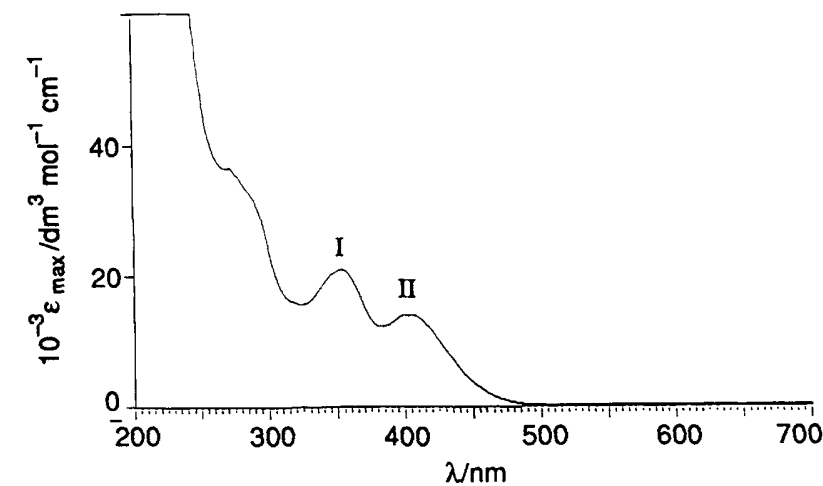

Fig. 4 Room-temperature electronic absorption spectrum of $\left[\mathrm{Pt}_{2}\right.$ $\left.(\mathrm{dppm})_{2}\left(\mathrm{PPh}_{3}\right)_{2}\right]\left[\mathrm{PF}_{6}\right]_{2}$ in acetonitrile
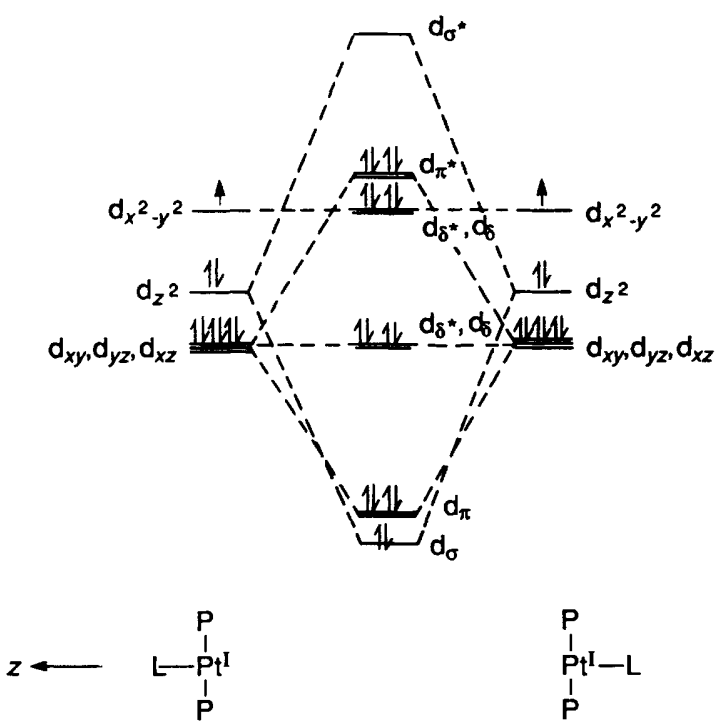

Fig. 5 A qualitative molecular orbital diagram for the $\left[\mathrm{Pt}^{\mathbf{1}}{ }_{2}\right.$ $\left.(\mathrm{dppm})_{2} \mathrm{X}_{2}\right]^{2+}$ complexes

destabilized by the interaction with four strongly donating anionic ligands, while the $\delta$ orbitals in diplatinum(1) systems such as $\left[\mathrm{Pt}_{2}(\mathrm{dppm})_{2}\left(\mathrm{PPh}_{3}\right)_{2}\right]^{2+}$ only interact with two dppm ligands. Hence the $\mathrm{d}_{\pi^{*}}-\mathrm{d}_{\delta}$ splitting in the $\mathrm{Pt}^{\mathrm{III}}-\mathrm{Pt}^{\mathrm{III}}$ must be smaller than that in $\mathrm{Pt}^{\mathrm{i}}-\mathrm{Pt}^{\mathrm{I}}$ systems.

When the axial ligands are pyridine, quinoline, $4 \mathrm{Bu}^{\mathrm{t}}$-py or mim the visible region of the absorption spectra is dominated by a broad tailing with low-to-moderate absorptivity. Fig. 6 shows the room-temperature absorption spectrum of an acetonitrile solution of $\left[\mathrm{Pt}_{2}(\mathrm{dppm})_{2}(\mathrm{py})_{2}\right]\left[\mathrm{PF}_{6}\right]_{2}$. The band around 350$400 \mathrm{~nm}$ is weak $\left(\varepsilon=10^{2} \mathrm{dm}^{3} \mathrm{~mol}^{-1} \mathrm{~cm}^{-1}\right)$. The spectrum of $\left[\mathrm{Pt}_{2}(\mathrm{dppm})_{2}(\mathrm{mim})_{2}\right]\left[\mathrm{PF}_{6}\right]_{2}$ (not shown), having the shortest $\mathrm{Pt}-\mathrm{Pt}$ distance, shows similar tailing with moderate absorptivity ( $\left.\varepsilon=10^{3} \mathrm{dm}^{3} \mathrm{~mol}^{-1} \mathrm{~cm}^{-1}\right)$ and there is virtually no absorption at $\lambda>400 \mathrm{~nm}$. It is therefore clear that the ${ }^{1}\left(\mathrm{~d}_{\pi^{*}} \longrightarrow \mathrm{d}_{\sigma^{*}}\right)$ transition is blue shifted in the case of $\left[\mathrm{Pt}_{2}(\mathrm{dppm})_{2} \mathrm{~L}_{2}\right]^{2+}$ (this is further confirmed by the excitation spectra of these complexes, see below). The blue shift is explained by the short $\mathrm{Pt}-\mathrm{Pt}$ separations in these complexes, leading to a greater $d_{\sigma}-d_{\sigma^{*}}$ splitting. It is important to characterize the crystal structure of $\left[\mathrm{Pt}_{2}(\mathrm{dppm})_{2}\left(\mathrm{PPh}_{3}\right)_{2}\right]^{2+}$ in order to verify the above argument. However, attempts to obtain suitable crystals have been unsuccessful. Nevertheless, it is known that the $R h-R h$ distance of $\left[\mathrm{Rh}_{2}\left(\mathrm{O}_{2} \mathrm{CMe}\right)_{2}\left(\mathrm{PPh}_{3}\right)_{2}\right][2.449(2) \AA]$ is considerably longer than that of $\left[\mathrm{Rh}_{2}\left(\mathrm{O}_{2} \mathrm{CMe}\right)_{4}(\mathrm{py})_{2}\right][2.227(3) \AA]{ }^{11}$ It is reasonable to expect $\mathrm{Pt}^{1}-\mathrm{Pt}^{1}$ complexes to display similar behaviour. Besides, the low-energy transitions of $\left[\mathrm{Pt}_{2}-\right.$ $\left.(\mathrm{dppm})_{2}\left(\mathrm{PPh}_{3}\right)_{2}\right]^{2+}$ are more intense than for those complexes with axial nitrogen bases. This may be explained by the socalled 'phosphine effect'. ${ }^{15}$ The complex $\left[\mathrm{Pt}_{2}(\mathrm{dppm})_{2}(\text { quin })_{2}\right]^{2+}$

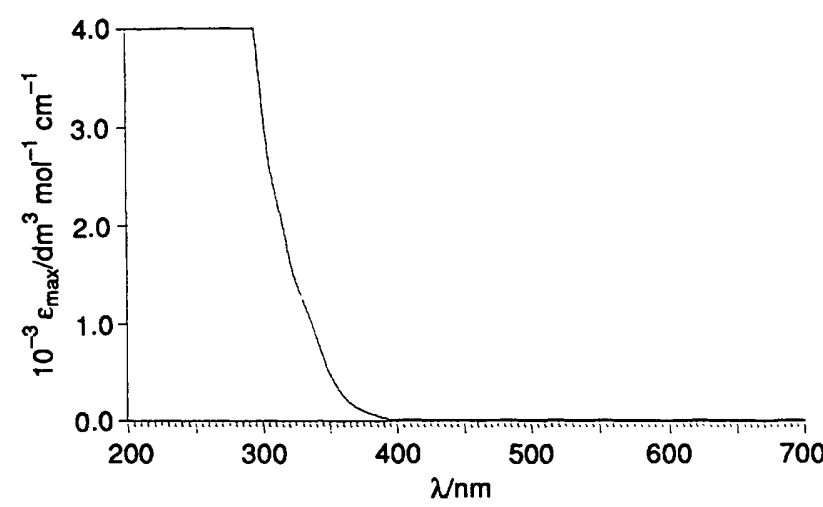

Fig. 6 Room-temperature electronic absorption spectrum of $\left[\mathrm{Pt}_{2}\right.$ $\left.(\mathrm{dppm})_{2}(\mathrm{py})_{2}\right]\left[\mathrm{PF}_{6}\right]_{2}$ in acetonitrile

displays an intense absorption at about $320 \mathrm{~nm}\left(\varepsilon=1.2 \times 10^{4}\right.$ $\mathrm{dm}^{3} \mathrm{~mol}^{-1} \mathrm{~cm}^{-1}$ ) and this is assigned to the intraligand $\pi-\pi^{*}$ transitions of quinoline. Although there are no distinct absorption bands in the visible region, the presence of metal-to-ligand charge-transfer transitions in this complex cannot be excluded. Two fairly distinguishable absorption bands in visible region are found for the complex $\left[\mathrm{Pt}_{2}(\mathrm{dppm})_{2}(\mathrm{tmpy}) \mathrm{Cl}\right]^{+}$. The intense absorption at $344 \mathrm{~nm}$ is assigned to the ${ }^{1}\left(d_{5 / \sigma^{*}} \longrightarrow d_{\sigma^{*}}\right)$ transition based on the expected insensitivity of the energy of the $\delta$ orbital towards the nature of axial ligand. The weak absorption around $400 \mathrm{~nm}\left(\varepsilon=200 \mathrm{dm}^{3} \mathrm{~mol}^{-1} \mathrm{~cm}^{-1}\right)$ should arise from the ${ }^{1}\left(\mathrm{~d}_{\pi^{*}} \longrightarrow \mathrm{d}_{\mathrm{\sigma}^{*}}\right)$ transition.

All the complexes mentioned as solids display photoluminescence at room temperature and at $77 \mathrm{~K}$. Figs. 7 and 8 show the emission spectra at room temperature and $77 \mathrm{~K}$ of $\left[\mathrm{Pt}_{2}(\mathrm{dppm})_{2}-\right.$ $\left.\left(\mathrm{PPh}_{3}\right)_{2}\right]\left[\mathrm{PF}_{6}\right]_{2}$ and $\left[\mathrm{Pt}_{2}(\mathrm{dppm})_{2}(\mathrm{py})_{2}\right]\left[\mathrm{PF}_{6}\right]_{2}$, respectively. The data are summarized in Table 5. Except for $\left[\mathrm{Pt}_{2}(\mathrm{dppm})_{2^{-}}\right.$ (quin) $]^{2+}$ which displays two emission bands, all the complexes exhibit a broad band centred around $600 \mathrm{~nm}$ but with no vibronic feature even at $77 \mathrm{~K}$. The long emission lifetimes (Table 5) and large Stoke's shift of the emission from the lowest-allowed absorption band indicate the phosphorescent nature of the emission. Upon cooling to $77 \mathrm{~K}$ all emission bands are intensified and become narrower and the maxima are slightly shifted to lower energy. Analogous to the $\left[\mathrm{Pt}_{2}-\right.$ $\left.\left(\mathrm{HPO}_{4}\right)_{4} \mathrm{~L}_{2}\right]^{n-}$ complexes, ${ }^{7}$ the temperature dependence of the half-width of the emission of the present complexes is not as large as that reported for the $d_{\sigma} \longrightarrow d_{\sigma^{*}}$ emission band of $\left[\operatorname{Re}_{2}(\mathrm{CO})_{6}(\mathrm{dmpm})_{2}\right] \cdot{ }^{16}$ For example, the half-height bandwidth of the emission of $\left[\mathrm{Pt}_{2}(\mathrm{dppm})_{2}(\mathrm{py})_{2}\right]^{2+}$ only increases by $17 \%$ when the temperature rises from 77 to $298 \mathrm{~K}$. We assign the emission to the ${ }^{3}\left(\mathrm{~d}_{\sigma^{*}} \longrightarrow \mathrm{d}_{\pi^{*}}\right)$ transition. The emission spectra of $\left[\mathrm{Pt}_{2}(\mathrm{dppm})_{2}(\text { quin })_{2}\right]\left[\mathrm{PF}_{6}\right]_{2}$ at room temperature and at $77 \mathrm{~K}$ are depicted in Fig. 9. At room temperature the spectrum consists of two emissions at 473 and $624 \mathrm{~nm}$ with the latter much more intense. The lifetime of the high-energy emission is too short to be measured by our instrument $(<20 \mathrm{~ns})$ while the lifetime of the low-energy emission is relatively long. At $77 \mathrm{~K}$ the spectrum is dominated by a high-energy vibronic emission and a relatively less-intense structureless one at $650 \mathrm{~nm}$. The observed $1391 \mathrm{~cm}^{-1}$ vibrational progression is consistent with the assignment of the high-energy emission to the fluorescence of quinoline. The emission at $650 \mathrm{~nm}$ is exceptionally lower in energy than the emission of $\left[\mathrm{Pt}_{2}(\mathrm{dppm})_{2}(\mathrm{py})_{2}\right]^{2+}$ or $\left[\mathrm{Pt}_{2}-\right.$ $\left.(\mathrm{dppm})_{2}(\mathrm{mim})_{2}\right]^{2+}$. This suggests that it may arise from a metalto-ligand charge transfer $(\mathrm{Pt} \rightarrow$ quin) or a ligand-field excited state. To clarify the origin of this emission, however, further spectroscopic work needs to be done.

For all the $\mathrm{Pt}^{1}-\mathrm{Pt}^{1}$ compounds mentioned, the excitation spectra become intensified with maxima slightly shifted to higher energy and reduction of band width upon cooling from 298 to $77 \mathrm{~K}$. Figs. 10 and 11 show the excitation spectra of the solid-state emissions of $\left[\mathrm{Pt}_{2}(\mathrm{dppm})_{2}\left(\mathrm{PPh}_{3}\right)_{2}\right]\left[\mathrm{PF}_{6}\right]_{2}$ and $\left[\mathrm{Pt}_{2}-\right.$ 
Table 5 Emission data for the Pt'-Pt' complexes at 298 and $77 \mathrm{~K}$

$\begin{array}{llll} & \begin{array}{l}\text { Excitation } \\ \text { maxima/nm } \\ \text { at } 298 / 77 \mathrm{~K}\end{array} & \begin{array}{l}\text { Emission } \\ \text { maxima/nm } \\ \text { at } 298 / 77 \mathrm{~K}\end{array} & \begin{array}{l}\text { Emission } \\ \text { lifetime }(\mu \mathrm{s}) \\ \text { at } 298 \mathrm{~K}\end{array} \\ \mathrm{Complex} & 422 / 398 & 589 / 570 & 2.8 \\ {\left[\mathrm{Pt}_{2}(\mathrm{dppm})_{2}\left(\mathrm{PPh}_{3}\right)_{2}\right]\left[\mathrm{PF}_{6}\right]_{2}} & 370 / 340 & 605 / 614 & 3.1 \\ {\left[\mathrm{Pt}_{2}(\mathrm{dppm})_{2}(\mathrm{py})_{2}\right]\left[\mathrm{PF}_{6}\right]_{2}} & 398 / 385 & 473,624 / & <2 \times 10^{-2},{ }^{n} \\ {\left[\mathrm{Pt}_{2}(\mathrm{dppm})_{2}(\mathrm{quin})_{2}\right]\left[\mathrm{PF}_{6}\right]_{2}} & & 491,527,650 & 3.9^{b} \\ {\left[\mathrm{Pt}_{2}(\mathrm{dppm})_{2}(\mathrm{mim})_{2}\right]\left[\mathrm{PF}_{6}\right]_{2}} & 368 / 352 & 577 / 601 & 3.2 \\ {\left[\mathrm{Pt}_{2}(\mathrm{dppm})_{2}(\mathrm{tmpy}) \mathrm{Cl}_{2}\right] \mathrm{PF}_{6}} & 405 / 347,396 & 591 / 601 & 3.2 \\ {\left[\mathrm{Pt}_{2}(\mathrm{dppm})_{2}\left(4 \mathrm{Bu}-\mathrm{pyy}_{2}\right]\left[\mathrm{PF}_{6}\right]_{2}\right.} & 345 / 339 & 600 / 609 & 2.1\end{array}$

${ }^{a}$ Lifetime of the emission at $473 \mathrm{~nm} .{ }^{b}$ Lifetime of the emission at $624 \mathrm{~nm}$.

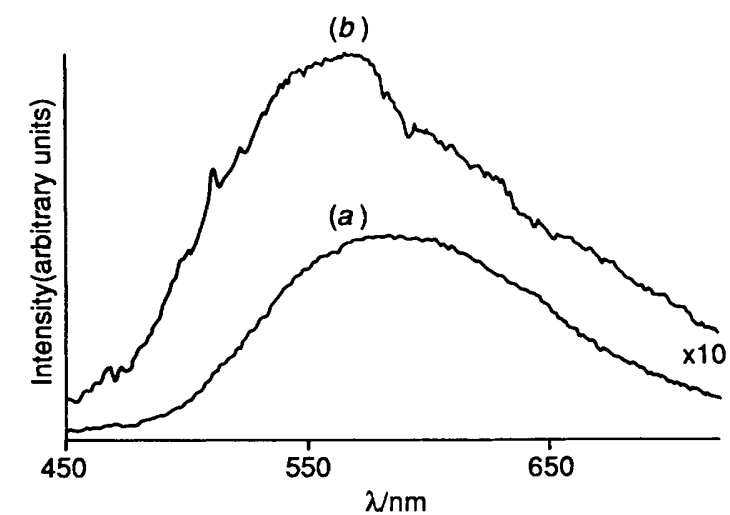

Fig. 7 Solid-state emission spectra of $\left[\mathrm{Pt}_{2}(\mathrm{dppm})_{2}\left(\mathrm{PPh}_{3}\right)_{2}\right]\left[\mathrm{PF}_{6}\right]_{2}$ at $298(a)$ and $77 \mathrm{~K}(b)$

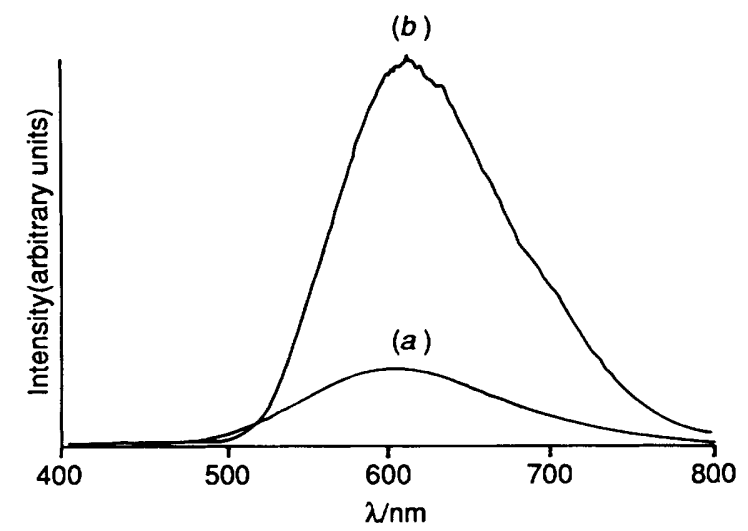

Fig. 8 Solid-state emission spectra of $\left[\mathrm{Pt}_{2}(\mathrm{dppm})_{2}(\mathrm{py})_{2}\right]\left[\mathrm{PF}_{6}\right]_{2}$ at 298 (a) and $77 \mathrm{~K}(b)$

$\left.(\mathrm{dppm})_{2}(\mathrm{py})_{2}\right]\left[\mathrm{PF}_{6}\right]_{2}$, respectively. When the temperature is changed from 298 to $77 \mathrm{~K}$ the excitation maximum of $\left[\mathrm{Pt}_{2}-\right.$ $\left.(\mathrm{dppm})_{2}\left(\mathrm{PPh}_{3}\right)_{2}\right]\left[\mathrm{PF}_{6}\right]_{2}$ shifts from 422 to $398 \mathrm{~nm}$ with a reduction in half-height bandwidth to $2908 \mathrm{~cm}^{-1}$, while the excitation maximum of $\left[\mathrm{Pt}_{2}(\mathrm{dppm})_{2}(\mathrm{py})_{2}\right]\left[\mathrm{PF}_{6}\right]_{2}$ shifts from 370 to $340 \mathrm{~nm}$ and the half-height bandwidth is reduced to 1961 $\mathrm{cm}^{-1}$. These features are consistent with the assignment of excited states produced by populating a $d_{\sigma^{*}}$ orbital. ${ }^{17}$ The good correlation of the excitation and absorption maxima of the complex $\left[\mathrm{Pt}_{2}(\mathrm{dppm})_{2}\left(\mathrm{PPh}_{3}\right)_{2}\right]^{2+}$ supports the assignment of the emissive state to ${ }^{3}\left(\mathrm{~d}_{\pi^{*}} \mathrm{~d}_{\sigma^{*}}\right)$. Besides, the excitation spectrum of $\left[\mathrm{Pt}_{2}(\mathrm{dppm})_{2}\left(\mathrm{PPh}_{3}\right)_{2}\right]\left[\mathrm{PF}_{6}\right]_{2}$ displays considerable intensity around $350 \mathrm{~nm}$ suggesting that, apart from the lowest-energy ${ }^{1}\left(d_{\pi^{*}} \longrightarrow d_{\sigma^{*}}\right)$ transition, the ${ }^{1}\left(d_{\delta^{*}} \longrightarrow d_{\sigma^{*}}\right)$ transition also gives rise to the emission. Since the emission of the complex is well fitted to a single-exponential first-order decay kinetics, the existence of dual emission is excluded. It is therefore reasonable to assign this behaviour to an efficient coupling between $\left(\mathrm{d}_{\delta^{*}} \mathrm{~d}_{\sigma^{*}}\right)$

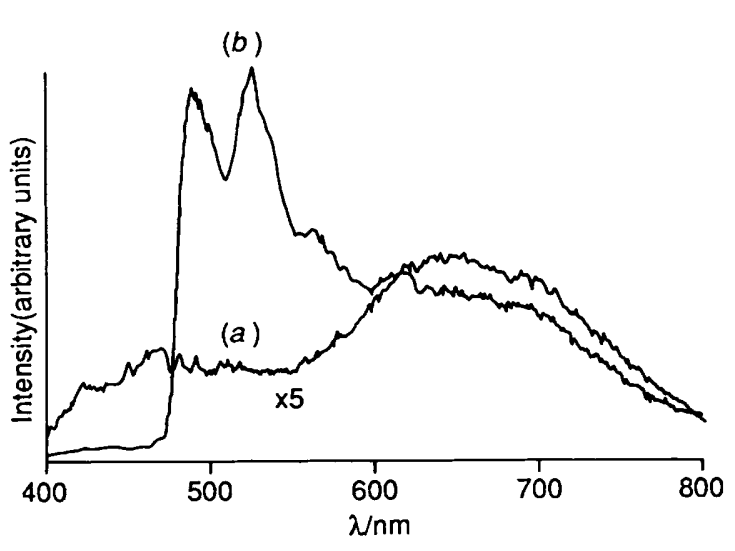

Fig. 9 Solid-state emission spectra of $\left[\mathrm{Pt}_{2}(\mathrm{dppm})_{2}(\text { quin })_{2}\right]\left[\mathrm{PF}_{6}\right]_{2}$ at $298(a)$ and $77 \mathrm{~K}(b)$

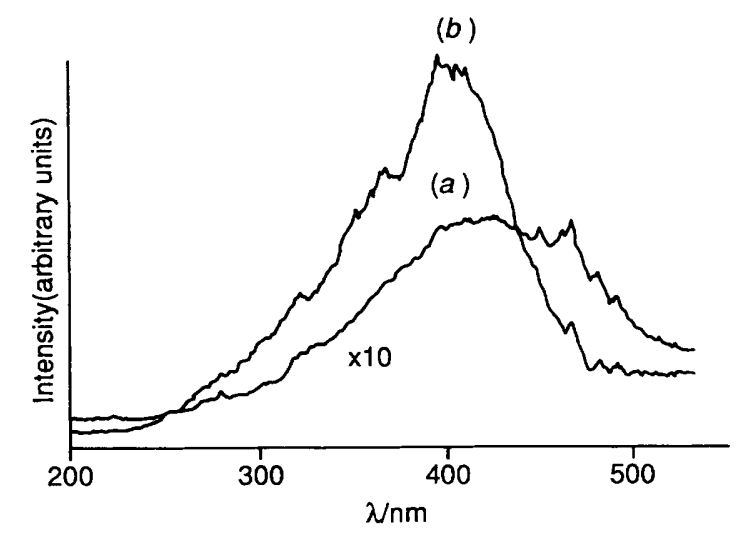

Fig. 10 Excitation spectra of $\left[\mathrm{Pt}_{2}(\mathrm{dppm})_{2}\left(\mathrm{PPh}_{3}\right)_{2}\right]\left[\mathrm{PF}_{6}\right]_{2}$ with emission monitored at $600 \mathrm{~nm}$ at $298 \mathrm{~K}(a)$ and $77 \mathrm{~K}(b)$

and $\left(d_{\pi^{*}} \cdot d_{\sigma^{*}}\right)$ excited states. The excitation spectrum of $\left[\mathrm{Pt}_{2}-\right.$ $\left.(\mathrm{dppm})_{2}(\mathrm{py})_{2}\right]\left[\mathrm{PF}_{6}\right]_{2}$ helps to locate the position of the ${ }^{1}\left(\mathrm{~d}_{\pi^{*}} \longrightarrow \mathrm{d}_{\sigma^{*}}\right)$ transition which is at about $370 \mathrm{~nm}$ at room temperature. Similarly, the excitation spectra of other $\mathrm{Pt}^{\mathrm{I}}-\mathrm{Pt}^{\mathrm{I}}$ complexes also span a large spectral region.

\section{Conclusion}

This work demonstrates a new class of inorganic luminescent materials containing a single metal-metal bond. The important feature of these complexes is that they undergo facile substitution and this allows complexes with various axial ligands to be obtained readily. The formation of the mixed-ligand complex $\left[\mathrm{Pt}_{2}(\mathrm{dppm})_{2}(\mathrm{tmpy}) \mathrm{Cl}\right]^{+}$with the sterically bulky ligand tmpy suggests it may be possible to construct complexes with two different types of axial ligands (for example donor and acceptor). Besides, $\mathrm{Pt}^{\mathrm{l}}-\mathrm{Pt}^{\mathrm{I}}$ complexes are long known to bind 
(b)

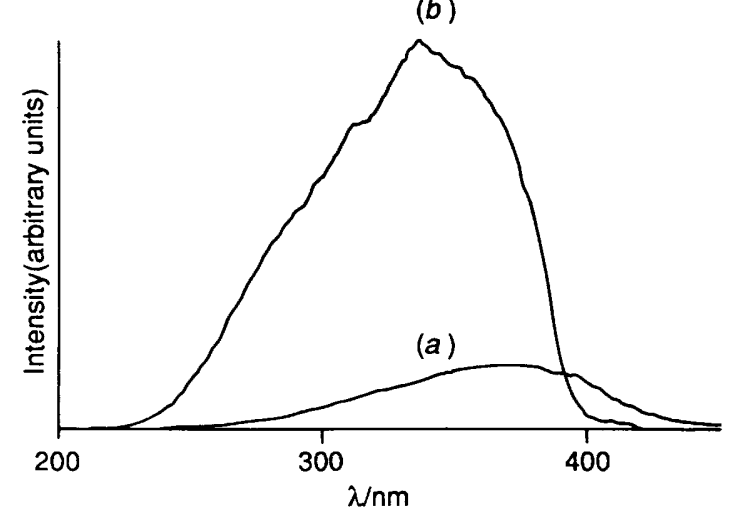

Fig. 11 Excitation spectra of $\left[\mathrm{Pt}_{2}(\mathrm{dppm})_{2}(\mathrm{py})_{2}\right]\left[\mathrm{PF}_{6}\right]_{2}$ with emission monitored at $600 \mathrm{~nm}$ at $298(a)$ and $77 \mathrm{~K}(b)$

small molecules like $\mathrm{CO}$ and $\mathrm{SO}_{2}{ }^{18}$ to form A-frame complexes. It should be noted that the formal oxidation state of platinum changes from I to II after binding with molecules like $\mathrm{CO}$. The entirely different photophysical properties of $\mathrm{Pt}^{\mathrm{I}}-\mathrm{Pt}^{1}$ and $\mathrm{Pt}^{\mathrm{II}}-\mathrm{Pt}^{\mathrm{II}}$ complexes are apparent. It is therefore interesting to investigate any changes of luminescent properties occurring after the binding reaction. This may point to the application of $\mathrm{Pt}^{1}-\mathrm{Pt}^{\mathrm{I}}$ complexes in the development of new types of luminescent chemical sensor. Work along these lines is now being undertaken.

\section{Acknowledgements}

We acknowledge financial support from the Croucher Foundation, the University of Hong Kong, Hong Kong Research Grants Council and the National Science Council (NSC) of Taiwan. C.-M. C. is grateful for a visiting professorship administered by the National Taiwan University, H.-K. Y. for a Croucher Studentship, administered by the Croucher Foundation of Hong Kong.

\section{References}

1 G. L. Geoffroy and M. S. Wrighton, Organometallic Photochemistry, Academic Press, New York, 1979.
2 See, for example, (a) V. M. Miskowski, W. P. Schaefer, B. Sadeghi, B. D. Santarsiero and H. B. Gray, Inorg. Chem., 1984, 23, 1154; (b) K.-S. Shin, R. J. H. Clark and J. K. Zink, J. Am. Chem. Soc., 1989, 111, 4244; (c) V. M. Miskowski, R. F. Dallinger, G. G. Christoph, D. E. Morris, G. H. Spies and W. H. Woodruff, Inorg. Chem., 1987, 26, 2127.

3 (a) C. M. Che, W.-M. Lee, T. C. W. Mak and H. B. Gray, J. Am. Chem. Soc., 1986, 108, 4446; (b) C. M. Che, T. C. W. Mak, V. M. Miskowski and H. B. Gray, J. Am. Chem. Soc., 1986, 108, 7840; (c) C. M. Che, L. G. Butler, P. J. Grunthaner and H. B. Gray, Inorg. Chem., 1985, 24, 4662; (d) M. Kurmoo and R. J. H. Clark, Inorg. Chem., 1985, 24, 4420 .

4 M. S. Wrighton and D. S. Ginley, J. Am. Chem. Soc., 1975, 97, 2065.

5 A. E. Stiegman, V. M. Miskowski and H. B. Gray, J. Am. Chem. Soc., 1986, 108, 2781.

6 S. J. Miller, M. P. Castellani, T. J. R. Weakley, D. R. Tyler, V. M. Miskowski and A. E. Stiegman, J. Phys. Chem., 1990, 94, 6599.

7 Y. K. Shin, V. M. Miskowski and D. G. Nocera, Inorg. Chem., 1990, 29, 2308.

8 J. I. Dulebohm, D. L. Ward and D. G. Nocera, J. Am. Chem. Soc., $1990,112,2969$.

9 (a) L. Manojlović-Muir, K. W. Muir and T. Solomun, J. Organomet. Chem., 1979, 179, 479; (b) L. Manoljlović-Muir, K. W. Muir and T. Solomun, Acta Crystallogr., Sect. B, 1979, 35, 237; (c) J. R. Fisher, A. J. Mills, S. Sumner, M. P. Brown, M. A. Thomson, R. J. Puddephatt, A. A. Frew, L. Manojlović-Muir and K. W. Muir, Organometallics, 1982, 1, 1421; $(d)$ M. N. I. Klan, C. King, J.-C. Wang, S. Wang and J. P. Fackler, jun., Inorg. Chem., 1986, 4656.

10 M. P. Brown, S. J. Franklin, R. J. Puddephatt, M. A. Thomson and K. R. Seddon, J. Organomet. Chem., 1979, 178, 281.

11 F. A. Cotton and T. R. Felthouse, Inorg. Chem., 1981, 20, 600.

12 L. E. Manzer and G. W. Parshall, Inorg. Chem., 1976, 15, 314.

13 D. P. Bancroft, F. A. Cotton, L. R. Falvello, S. Hans and W. Schwotzer, Inorg. Chim. Acta, 1984, 87, 147.

14 R. A. Newman, D. S. Martin, R. F. Dallinger, W. H. Woodruff, A. E. Stiegman, C. M. Che, W. P. Schaefer, V. M. Miskowski and H. B. Gray, Inorg. Chem., 1991, 30, 4647.

15 V. M. Miskowski, J. L. Robbin, G. S. Hammond and H. B. Gray, J. Am. Chem. Soc., 1976, 98, 2477.

16 A. E. Stiegman and V. M. Miskowski, J. Am. Chem. Soc., 1988, 110, 4053.

17 (a) R. A. Levenson and H. B. Gray, J. Am. Chem. Soc., 1975, 97, 6042; (b) V. M. Miskowski, T. P. Smith, T. M. Loehr and H. B. Gray, J. Am. Chem. Soc., 1985, 107, 7925.

18 M. P. Brown, J. R. Fisher, R. J. Puddephatt and K. R. Seddon, Inorg. Chem., 1979, 18, 2808.

Received 3rd June 1992; Paper 2/02930H 